\title{
Hydrodynamic stability of a sheared liquid film
}

\author{
By ROB MIESEN ${ }^{1} \dagger$ AND BENDIKS JAN BOERSMA ${ }^{2} \ddagger$ \\ ${ }^{1}$ Koninklijke/Shell-Laboratorium, Amsterdam, Postbus 38000, 1030 BN Amsterdam, \\ The Netherlands \\ ${ }^{2}$ University of Twente, Postbus 217, 7500 AE Enschede, The Netherlands
}

(Received 29 November 1993 and in revised form 22 May 1995)

We study the hydrodynamic stability of a thin layer of liquid that is sheared by a gas. First, the interface conditions for the free surface approximation of the problem are discussed. We then study the stability of the flow to disturbances with phase speeds smaller than the maximum velocity in the liquid film, i.e. the internal mode, extending previous results and resolving some apparent contradictions.

The dynamic effect of the gas is studied by dropping the free surface approximation and solving the Orr-Sommerfeld equation for the gas together with that for the liquid. The effect on the stability of the liquid film is very large, which is explained by the fact that the imaginary part of the wave speed (which determines the stability of the film) is very small. Consequently the free surface approximation is, in general, not correct.

We then study the dependence of the critical Reynolds number on the Weber number, on the curvature of the liquid velocity profile and on the properties of the gas. With the gas included, a second mode of instability is found which has a phase velocity that is, in general, larger than the maximum liquid velocity and corresponds to capillary-gravity waves. We compare results with experiments from the literature; good agreement is found. Finally, a suggestion on the relevance of this study to the generation of 'roll waves', which are important from a practical point of view, is given.

\section{Introduction}

The hydrodynamic stability of thin liquid films, bounded by a wall and sheared by a gas, plays an important role in many industrial processes. A good example is the flow of hydrocarbons in furnace tubes, condensate in gas wells and liquid in cooling systems of nuclear installations. The liquid flows as an annular film along the wall of the vertical pipes, while the gaseous components flow in the centre of the tubes. The stability theory of such liquid films may be used to predict the transition to dispersed flow, where liquid droplets are entrained by the gas.

In this report we will consider the hydrodynamic stability of a thin liquid film that is bounded by a wall, and which is moving due to the shear force of a gas flowing over its surface and gravity. Several authors have considered this or a similar problem, often originating from the study of the generation of water waves by wind and we

$\dagger$ Present address: Shell Internationale Petroleum Maatschappij, Postbus 162, 2501 AN Den Haag, The Netherlands.

$\ddagger$ Present address: Laboratory for Aero- and Hydrodynamics, Rotterdamseweg 145, 2628 AL Delft, The Netherlands. 


\begin{tabular}{ccc} 
Quantity & Liquid & Gas \\
Mass density $\rho\left(\mathrm{kg} \mathrm{m}^{-3}\right)$ & $10^{3}$ & 1 \\
Dynamic viscosity $\mu(\mathrm{mPa} \mathrm{s})$ & $1-10$ & 0.01 \\
Velocity $U\left(\mathrm{~m} \mathrm{~s}^{-1}\right)$ & 1 & $10-100$ \\
Length scale $d(\mathrm{~m})$ & $10^{-4}-10^{-3}$ & $\geqslant 0.01$ \\
Reynolds number $\rho U d / \mu$ & 100 & $\geqslant 10^{4}$ \\
Interfacial tension $\sigma\left(\mathrm{N} \mathrm{m}^{-1}\right)$ & \multicolumn{2}{c}{0.05}
\end{tabular}

TABLE 1. Characteristic values of the important physical parameters.

will briefly discuss the more important papers with respect to this study. But first, to set the scene, we give some characteristic values of the relevant parameters for the type of flow of interest (table 1). The mass density and the dynamic viscosity of the liquid are much larger than those of the gas, but the kinematic viscosity $v=\mu / \rho$ can be of the same order of magnitude. A typical gas velocity is at least an order of magnitude larger than the velocity of the liquid and while the liquid film can be considered laminar, the gas flow will be turbulent.

Miles (1962b) distinguishes four mechanisms for the generation of waves on the surface of a liquid. He argues that for thin films the formation of long waves will be inhibited by wall friction and that therefore only short waves will be formed. For short waves the important mechanism for energy transfer from the flow to the waves will be through the viscous Reynolds stress. For longer waves energy transfer is through the well known mechanism of inviscid Reynolds stress in the critical layer (where the wave speed equals the mean gas speed) of a curved velocity profile, e.g. Miles (1957, 1959). The essential difference between these two mechanisms is in the position of the critical layer: in the viscous sublayer for short waves (where the velocity profile is linear) and outside the viscous sublayer for long waves. For short waves two different cases have to be considered: waves that move faster than the maximum velocity in the liquid and waves that are slower than this velocity.

In the first case the critical layer is in the gas and energy transfer is through the viscous Reynolds stress in the immediate neighbourhood of the surface. This case has been considered by, for example, Miles (1962a) and Cohen \& Hanratty (1965) following the 'quasi static' approximation or 'divided attack' as Benjamin (1959) calls it: first the stresses that a gas exerts on a solid wavy surface are calculated and then the equations of motion for the liquid are solved, the calculated stresses entering through the conditions for the liquid flow at the gas/liquid interface.

The second case is considered by Miles (1960) and by Smith \& Davis (1982) $\dagger$. In this case, which Miles suggests is important for very thin films, the critical layer will be in the liquid and energy transfer is through the viscous Reynolds stress in the liquid. He shows that the film can be unstable because the film is sheared alone, i.e. the film can be unstable even if the dynamic influence of the gas (in the form of stress variations on the liquid's surface) is negligible. However, Miles' (1960) asymptotic results are valid for disturbances that are longer than the thickness of the liquid layer only, as will be explained later. This conclusion can also be found

$\dagger$ The full problem for the gas and the liquid was also studied by Lock (1954) and Feldman (1957). However, their calculations are incorrect (Miles 1960; Blennerhassett 1980; Hooper \& Boyd 1983). Blennerhassett also studies the full problem, focusing on nonlinear waves. This makes several assumptions necessary that are not correct in the present context. 
in the paper by Smith \& Davis (1982), who solve the problem numerically. It is illustrated by their figure 2 (compare the curves $b$ and $c$ ). However, the rest of their calculations use interface conditions that differ from those of Miles and which are incorrect to describe the stability of the flow in a liquid film that is sheared by a gas.

The paper by Craik (1966) should also be mentioned. In that paper the hydrodynamic stability of thin liquid films (typically thinner than $10^{-3} \mathrm{~m}$ ) is studied both experimentally and theoretically for relatively small Reynolds numbers of the liquid layer (typically smaller than 10). The experiments show the presence of two types of waves: 'fast waves', having wave speeds larger than the surface velocity of the liquid film, and 'slow waves', having wave velocities somewhat smaller than the surface velocity. The first were only present at a high enough Reynolds number of the film and are of the type considered by Miles (1962a) and Cohen \& Hanratty (1965). The 'slow waves' are present at low enough Reynolds number. Thus this instability is different from the one considered by Miles (1960), which would also lead to waves slower than the surface velocity but only at high enough Reynolds number. Miles does not find these slow waves from his analysis because he ignores surface stress fluctuations of the gas and considers large Reynolds numbers only.

The same problem as formulated by Miles (1960) is considered here and a minor error in that paper will be corrected. Furthermore, the analysis will be extended to include larger wavenumbers, which have been shown to be important (Smith \& Davis 1982). The discrepancy between the curves of neutral stability of the flow as found by Miles and as found by Smith \& Davis will be clarified. In the second part of this paper the dynamic effect of the gas on the stability of the liquid film is considered and consequently the 'interfacial mode' will enter the analysis (see Blennerhassett 1980 and Yiantsios \& Higgins 1988). Our results will be compared to previous results for that mode. Then, our results will be compared with experiments presented by Cohen \& Hanratty (1965) and Craik (1966); agreement is shown to be very good. Finally, the potential relevance of this study to the generation of 'roll waves' and entrainment will be discussed.

\section{The primary flow}

The annular flow in the liquid film is assumed to be laminar because its Reynolds number is not very large (see table 1). The liquid flow is driven by the shear force of the gas on the liquid interface and possibly by a pressure gradient or gravity. The flow of the gas in the pipe is turbulent and is driven by a pressure gradient in the direction of the pipe. We will use a 'quasi-steady' description of the gas flow, assuming the velocity of the primary flow in the gas to be given by its time-average and so ignoring turbulent fluctuations. However, when studying the stability of the flow we will admit time-dependent perturbations. A mechanism for the generation of waves by stress fluctuations has been studied by, for example, Phillips (1962). The justification of the above assumption is discussed by, for example, Miles (1962a) and Craik (1966) and is supported by the observation that liquid films can have a perfectly smooth surface in the presence of a turbulent gas flow (Cohen \& Hanratty 1965). Furthermore, it is possible to include turbulent effects partly by incorporating turbulent Reynolds stresses (with some closure model) in such a quasi-static model (see for instance van Duin \& Janssen 1992). 


\subsection{The liquid film}

The flow can be solved in a cylindrical geometry (Boomkamp \& Miesen 1992) but because it is assumed axisymmetric and the liquid film is sufficiently thin it can be considered two-dimensional (see e.g. Miesen et al. 1992 and figure 1). The flow $U(y)$ in the film along the plate in the $x$-direction can be found from the momentum balance:

$$
-P_{x}-g \rho_{l}+\mu_{l} \frac{\mathrm{d}^{2} U}{\mathrm{~d} y^{2}}=0,
$$

where $y$ is the distance to the plate, $P_{x}$ is the axial pressure gradient, $g$ is the gravitational acceleration in the negative $x$-direction, $\rho_{l}$ is the density of the liquid and $\mu_{l}$ its dynamic viscosity. As $P_{x}$ is independent of $y$ (see e.g. Yih 1967), equation (2.1) can be integrated directly to give $U(y)$. The integration constants are determined by conditions at the wall and at the liquid/gas interface. No-slip at the wall gives $U(-d)=0$. Continuity of shear stress across the interface gives the condition $\mu_{l} \mathrm{~d} U / \mathrm{d} y=\tau$ at $y=0$, where $\tau$ is the shear force that the gas exerts on the liquid interface. Integrating (2.1) then gives

$$
\mu_{l} U(y)=\frac{1}{2}\left(P_{x}+g \rho_{l}\right)\left(y^{2}-d^{2}\right)+(y+d) \tau .
$$

We may introduce a dimensionless coordinate and velocity as

$$
\tilde{y}=\frac{y}{d}, \quad \tilde{U}=\frac{\mu_{l} U}{d \tau}=\frac{U}{U_{i}} .
$$

Here, $U_{i}=d \tau / \mu_{l}$ is the liquid velocity at the interface if the velocity profile is linear, i.e. if $P_{x}+g \rho_{l}$ is negligible. Substituting (2.3) into (2.2) and dropping tildes gives

$$
U(y)=A y^{2}+y+1-A, \quad A=\frac{\left(P_{x}+g \rho_{l}\right) d}{2 \tau} .
$$

Or in in a frame that moves at the interfacial speed,

$$
U(y)=A y^{2}+y, \quad-1 \leqslant y \leqslant 0 .
$$

From (2.4) it follows that if $A=0.75$ there is a net downward flow, indicating that the shear force exerted by the gas is not sufficient to transport the film upwards. For $A=0.5$ the shear stress at the wall is zero, corresponding to the onset of 'flow reversal' (see for example Hetstroni 1982). We have chosen the gas flow to be in the direction opposite to gravity. If the gas flow is downward the velocity profile in the liquid film is still given by (2.4), replacing $+g \rho_{l}$ by $-g \rho_{l}$. For pipes larger than, say, $0.01 \mathrm{~m}, P_{x}$ is usually of the order of or smaller than $g \rho_{l}$ so that $A$ can be negative. If the shear force $\tau$ is very large so that $A \ll 1$, the velocity profile is approximately linear and the stability problem is equivalent to that of Miles (1960), who considers a laminar horizontal film under zero pressure gradient.

We estimate the shear force of the gas from $\tau=\frac{1}{2} c_{f} \rho_{g} U_{g}^{2}$, with $c_{f}$ the friction factor which is of order 0.02 for the parameters in table 1 (see Wallis 1969), $\rho_{g}$ the mass density of the gas and $U_{g}$ the gas velocity. From the definition (2.4) of $A$ we then find that $A=0.5$ corresponds to $U_{g}=20 \mathrm{~m} \mathrm{~s}^{-1}$ and $A<0.1$ if $U_{g}>50 \mathrm{~m} \mathrm{~s}^{-1}$.

\subsection{The gas}

The gas flow is a developed turbulent flow in a pipe. The time-averaged velocity profile can be approximated by a linear velocity profile close to the film and the well-known logarithmic profile (e.g. Kays \& Crawford 1966) further away from the 


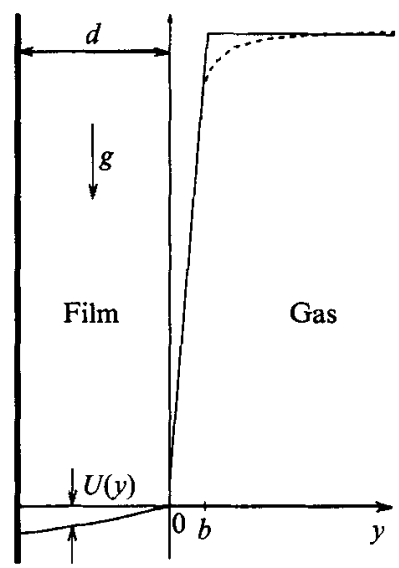

FIGURE 1. The flow of a liquid film that is sheared by a gas.

interface. A different profile is used in studies of wave generation by wind to which we will refer later, Miles (1962a):

$$
\begin{aligned}
U(y) & =\left(\tau / \mu_{g}\right) y, \\
U(y) & =\left(\tau / \rho_{g}\right)^{1 / 2}\left[s+\left(\gamma-\tanh \frac{1}{2} \gamma\right) / \kappa\right], \\
\sinh \gamma & =\frac{2 \kappa\left(\rho_{g} \tau\right)^{1 / 2}}{\mu_{g}}\left[y-s \mu_{g} /\left(\rho_{g} \tau\right)^{1 / 2}\right],
\end{aligned}
$$

with $\tau$ the shear stress, $\mu_{\mathrm{g}}$ the dynamic viscosity of the gas, $\kappa=0.4$ the Von Kármán constant (Hinze 1975) and $s$ determines the thickness of the viscous sublayer (often taken to be between 5 and 8 ). To asymptotically analyse or numerically efficiently solve the problem, the gas velocity profile is approximated further, as done by Yih (1990). The linear velocity profile of the viscous sublayer is extrapolated to the value at the pipe centre, after which the velocity is taken constant (figure 1). Note that this is correct only because we consider waves that have a critical layer in the viscous sublayer, so that velocity profile curvature is not important for the mechanism of instability (see the Introduction). Using (2.3) to make the profile dimensionless gives

$$
U(y)= \begin{cases}m y, & 0 \leqslant y \leqslant b \\ m b, & y \geqslant b\end{cases}
$$

with $m=\mu_{l} / \mu_{g}$ and the thickness $b$ of the shear layer in the gas defined by

$$
b=\frac{\mu_{g} U_{g}}{d \tau}=\frac{U_{g}}{m U_{i}},
$$

where $U_{\mathrm{g}}$ is the gas velocity with respect to the liquid/gas interface. Note that the tangential stress in the primary flow given by $(2.5)$ and $(2.7)$ is continuous across the interface.

\section{The stability problem}

\subsection{The Orr-Sommerfeld equation}

To study the stability of the above incompressible, parallel primary flow, the NavierStokes equations can be simplified to the Orr-Sommerfeld equation if the disturbances of the flow are assumed to be small, retaining only the linear terms in these distur- 
bances. Disturbances can then be written as (e.g. Drazin \& Reid 1986; Yih 1990)

$$
f(x, y, t)=\hat{f}(y) \exp [\mathrm{i} \alpha(x-c t)],
$$

where $f, \hat{f}, \alpha$, and $c$ stand for a disturbance, its $y$-dependent part, its wavenumber and its phase velocity. Note that $c$, as a function of $\alpha$ and the flow parameters, has to be determined from the following analysis. The real part of $c$ gives the velocity of the wave, while the imaginary part of $\alpha c$ is the growth rate, with $\operatorname{Im}(\alpha c)>0$ for growing waves. Writing the Orr-Sommerfeld equations in dimensionless form, using the characteristic length scale $d$, velocity $U_{i}$, time $d / U_{i}$, viscosity $\mu_{l}$, mass density $\rho_{l}$, and pressure $\rho_{l} U_{i}^{2}$, gives for the three regions defined by (2.5) and (2.6) or (2.7) (Yih 1967)

$$
\begin{gathered}
\left(\frac{\mathrm{d}^{2}}{\mathrm{~d} y^{2}}-\alpha^{2}\right)^{2} \chi(y)=\mathrm{i} \alpha R\left[\left(A y^{2}+y-c\right)\left(\frac{\mathrm{d}^{2}}{\mathrm{~d} y^{2}}-\alpha^{2}\right)-2 A\right] \chi(y), \quad-1<y<0, \\
\left(\frac{\mathrm{d}^{2}}{\mathrm{~d} y^{2}}-\alpha^{2}\right)^{2} \phi(y)=\mathrm{i} \alpha \operatorname{Rrm}(m y-c)\left(\frac{\mathrm{d}^{2}}{\mathrm{~d} y^{2}}-\alpha^{2}\right) \phi(y), \quad 0<y<b, \\
\left(\frac{\mathrm{d}^{2}}{\mathrm{~d} y^{2}}-\alpha^{2}\right)^{2} \psi(y)=\mathrm{i} \alpha \operatorname{Rrm}(m b-c)\left(\frac{\mathrm{d}^{2}}{\mathrm{~d} y^{2}}-\alpha^{2}\right) \psi(y), \quad y>b,
\end{gathered}
$$

where the Reynolds number $R=\rho_{l} U_{i} d / \mu_{l}, r=\rho_{g} / \rho_{l} ; \psi, \phi$, and $\chi$ are the $y$-dependent parts of the stream functions defined by, for example,

$$
\left.\begin{array}{l}
u(x, y, t)=U(y)+\frac{\partial \chi(y)}{\partial y} \exp [\mathrm{i} \alpha(x-c t)], \\
v(x, y, t)=-\mathrm{i} \alpha \chi(y) \exp [\mathrm{i} \alpha(x-c t)],
\end{array}\right\}
$$

where $u, v$ are the disturbances of the velocity in the $x$ - and $y$-directions.

\subsection{Boundary and interface conditions}

The no-slip and zero perpendicular velocity conditions at the wall give

$$
\chi(-1)=\chi^{\prime}(-1)=0,
$$

where a prime represents differentiation with respect to $y$. Furthermore, disturbances should be small far from the interface, so that

$$
\psi(\infty)=\psi^{\prime}(\infty)=0
$$

At the interface we use the continuity of the two velocity and stress components. The correct form of these continuity conditions is given by Yih (1990):

$$
\begin{array}{rlr}
\chi=\phi & \text { at } y & =0, \\
\chi^{\prime}-\phi^{\prime}=\chi(m-1) / c & \text { at } y & =0, \\
\chi^{\prime \prime}+\alpha^{2} \chi=m^{-1}\left(\phi^{\prime \prime}+\alpha^{2} \phi\right)-2 A \chi / c & \text { at } y & =0, \\
\chi^{\prime \prime \prime}-3 \alpha^{2} \chi^{\prime}+\mathrm{i} \alpha R\left(c \chi^{\prime}+\chi\right)-m^{-1}\left(\phi^{\prime \prime \prime}-3 \alpha^{2} \phi^{\prime}\right)-\mathrm{i} \alpha R r\left(c \phi^{\prime}+m \phi\right) & \\
-\mathrm{i} \alpha R\left(\sin \beta F+\alpha^{2} S\right) \chi / c=0 & \text { at } y & =0,
\end{array}
$$

where $\beta$ is the angle between the direction of gravity and the negative $y$-axis, $F=g\left(\rho_{l}-\rho_{g}\right) d /\left(\rho_{l} U_{i}^{2}\right)$ and $S=\sigma /\left(\rho_{l} U_{i}^{2} d\right)$, with $\sigma$ as the interfacial tension. In deriving these conditions $U(0)=0$ is used. 
It is important to note that (3.10) and (3.11) reduce to the conditions for a sheared liquid film with a free surface, as used by Miles (1960), if $r \rightarrow 0, m \rightarrow \infty$ and $m r \rightarrow 0$. Equations (3.8) and (3.9), i.e. continuity of the velocity, do not apply for a free surface. Thus, a gas/liquid interface can only be assumed to be a free surface if the density and the dynamic viscosity of the liquid are much larger than those of the gas, while the liquid's kinematic viscosity should be much smaller $(m r \rightarrow 0)$. For an air/water system at atmospheric pressure $m \approx 50$ and $r \approx 10^{-3}$, so that at least in this case the free surface conditions used by Miles seem to apply. If the gas is at a higher pressure, or if the liquid is much more viscous, $m r=O(1)$ and the free surface approximation does not hold. We will see, however, that even for an air/water system the dynamic influence of the gas is large.

Smith \& Davis (1982) have considered the same problem as Miles (1960), solving it numerically for all wavenumbers but with different conditions at the gas/liquid interface. The difference is due to the discontinuity in the tangential stress of the basic flow of Smith \& Davis, giving additional terms. For the flow considered here, i.e. a liquid that is sheared by a gas, the interface conditions as used by Miles should therefore be used $\dagger$. Note, however, that Smith \& Davis also perform one calculation with the equations used by Miles.

At the 'virtual interface' $y=b$ between the region in the gas where the velocity is assumed constant and the region where there is a velocity gradient, similar conditions as the above hold (Yih 1990):

$$
\begin{array}{cr}
\psi=\phi & \text { at } y=b, \\
\psi^{\prime}-\phi^{\prime}=m \psi /(c-m b) & \text { at } y=b, \\
\psi^{\prime \prime}+\alpha^{2} \psi=\phi^{\prime \prime}+\alpha^{2} \phi & \text { at } y=b, \\
\phi^{\prime \prime \prime}-3 \alpha^{2} \phi^{\prime}+\mathrm{i} \alpha \operatorname{Rrm}\left[(c-m b) \phi^{\prime}+m \phi\right]-\mathrm{i} \alpha \operatorname{Rrm}(c-m b) \psi^{\prime} \\
-\psi^{\prime \prime \prime}+3 \alpha^{2} \psi^{\prime}-2 m \alpha^{2} \psi /(c-m b)=0 & \text { at } y=b .
\end{array}
$$

Note that the last term of equation (3.15), was assumed to be zero by Yih (1990) with the argument that in fact the mean shear stress has no jump at $y=b$. Since this term is $O\left(R^{-1}\right)$ it can be neglected anyway in Yih's approximation. In the present paper we have also neglected this term and checked that differences are indeed small whether this term is included or not. If differences are not small it means that the approximation of the gas velocity profile by $(2.7)$ cannot be used. If the velocity profile (2.6) is used the last term in (3.15) is zero because the shear stress is continuous at $y=b$, and (3.14) could be argued to have an extra term because the curvature has a jump at $y=b$ which should again be small.

\section{A free surface and a linear velocity profile}

The simplest form of the stability problem is obtained by approximating the gas/liquid interface by a free surface for the liquid and using a linear velocity profile in the liquid. The conditions for the first assumption have been discussed in the previous section. The assumption of a linear velocity profile is correct if we consider like Miles (1960) a liquid film on a horizontal plate, or if the gas velocity in a vertical arrangement is very high so that $A \approx 0$ in (2.5). The stability of such a flow is described by equation (3.2) with $A=0$, with conditions (3.6) and the limiting form

$\dagger$ A more comprehensive discussion on the inferface conditions is available from the authors or the JFM Editorial Office. 
of (3.10) and (3.11):

$$
\begin{array}{lr}
\left(\frac{\mathrm{d}^{2}}{\mathrm{~d} y^{2}}-\alpha^{2}\right)^{2} \chi(y)=\mathrm{i} \alpha R(y-c)\left(\frac{\mathrm{d}^{2}}{\mathrm{~d} y^{2}}-\alpha^{2}\right) \chi(y) & \text { for }-1<y<0, \\
\chi=\chi^{\prime}=0 & \text { at } y=-1, \\
\chi^{\prime \prime}+\alpha^{2} \chi=0 & \text { at } y=0, \\
\varpi=\chi^{\prime \prime \prime}-3 \alpha^{2} \chi^{\prime}+\mathrm{i} \alpha R\left(c \chi^{\prime}+\chi\right)-\mathrm{i} \alpha R\left(\sin \beta F+\alpha^{2}\right) S \chi / c=0 & \text { at } y=0 .
\end{array}
$$

Equation (4.1) has four independent solutions, two of which can be seen to be given by:

$$
\chi_{1}(y)=\cosh [\alpha(y+1)], \quad \chi_{2}(y)=\sinh [\alpha(y+1)] .
$$

These solutions are called the inviscid solutions since they are solutions if $R \rightarrow \infty$. The two other solutions can be identified with the viscous boundary layers at the wall and at the interface.

\subsection{Miles}

Assuming that $\alpha R \gg 1$, Miles (1960) approximates these viscous solutions by

$$
\begin{gathered}
\chi_{3}(y)=(\alpha R)^{-2 / 3} \int_{\infty}^{(\alpha R)^{1 / 3}(y-c)}\left[(\alpha R)^{1 / 3}(y-c)-\zeta\right] \operatorname{Ai}\left(\mathrm{e}^{\mathrm{i} \pi / 6} \zeta\right) \mathrm{d} \zeta, \\
\chi_{4}(y)=(y-c)^{-5 / 4} \exp \left[\frac{2}{3}(\mathrm{i} \alpha R)^{1 / 2}(y-c)^{3 / 2}\right],
\end{gathered}
$$

for which he refers to Lin (1955); Ai() denotes the Airy-function of the first kind (see for instance Abramowitz \& Stegun 1965). The solution $\chi_{3}$ is associated with the boundary layer near the wall and is exponentially small at the interface if $(\alpha R)^{1 / 3} \gg 1$, and vice versa for $\chi_{4}$. The solution of (4.1) for the stream function $\chi$ is a linear combination of $\chi_{1,2,3,4}$, which is substituted into the conditions (4.2)-(4.4) giving four linear algebraic equations in the coefficients of $\chi_{1,2,3,4}$. Since $\chi_{3}$ and $\chi_{4}$ are exponentially small at the interface and the wall, respectively, Miles finds the following eigenvalue equation for $c$ :

$$
\left|\begin{array}{cccc}
\chi_{1 w} & \chi_{2 w} & \chi_{3 w} & 0 \\
\chi_{1 w}^{\prime} & \chi_{2 w}^{\prime} & \chi_{3 w}^{\prime} & 0 \\
\varpi_{1 i} & \varpi_{2 i} & 0 & \varpi_{4 i} \\
2 \alpha^{2} \chi_{1 i} & 2 \alpha^{2} \chi_{2 i} & 0 & \chi_{4 i}^{\prime \prime}+\alpha^{2} \chi_{4 i}
\end{array}\right|=0,
$$

where the first subscript identifies the individual solutions and the second the point of evaluation ( $w$ at the wall, and $i$ at the interface). Expanding this determinant and substituting (4.4) and the expressions for $\chi_{1,2,3,4}$, this equation can be written as (see the Appendix):

$$
\begin{gathered}
{\left[\alpha \operatorname{coth} \alpha+\frac{\alpha^{2} \operatorname{cosech}^{2} \alpha F(z)(c+1)}{1-\alpha(c+1) F(z) \operatorname{coth} \alpha}\right](c+2 \mathrm{i} \alpha / R)^{2}} \\
+(c+2 \mathrm{i} \alpha / R)-\alpha^{2} S+O\left(R^{-3 / 2}, S / R^{2}\right)=0,
\end{gathered}
$$

where we have used the definition of the Tietjens function:

$$
F(z)=1+\frac{\int_{\infty}^{-z} \zeta \operatorname{Ai}\left(\mathrm{e}^{\mathrm{i} \pi / 6} \zeta\right) \mathrm{d} \zeta}{z \int_{\infty}^{-z} \operatorname{Ai}\left(e^{\mathrm{i} \pi / 6} \zeta\right) \mathrm{d} \zeta}, \quad z=(\alpha R)^{1 / 3}(c+1) .
$$


Equation (4.9) for the eigenvalue $c$ of the problem is very similar to equation (4.6) of the paper by Miles (1960), except for an extra factor $[1-\alpha(c+1) F(z) \operatorname{coth} \alpha]^{-1}$ in the second term which is probably due to an error in Miles's approximation of (4.8). We could now approximate this equation further along the lines of Miles. Here we choose to solve equation (4.9) directly at the cost of some extra CPU timet. Comparing the results with the extra factor excluded or included gives that the critical Reynolds number for zero surface tension $(S=0)$ changes from 225 (with additional approximations Miles finds 203) to 136. This is considerably closer to the result 78.6 as found by Smith \& Davis (1982) (their result for the same boundary conditions as Miles). For $S=0.1$ these numbers are 787 (Miles) and 618 (equation (4.9)). Smith \& Davis do not give a result for $S=0.1$, but in the following 631 is found.

All other corrected results of Miles (1960) could now easily be calculated. Smith \& Davis show, however, that the neutral curve as calculated from equation (4.9) is not correct for $\alpha>1$. This is because the imaginary part of the phase velocity is small, say order $(\alpha R)^{-1 / 3}$, and the approximate solutions (4.6) and (4.7) introduce errors that are of the same order of magnitude or larger for $\alpha>1$.

\subsection{Improved solutions}

To improve on the solutions of (4.1) there are several options. An exact solution can be given (Feldman 1957), which is a linear combination of $\chi_{1,2}$ as defined in (4.5) and

$$
\begin{gathered}
\chi_{3}(y)=\frac{1}{\alpha} \int_{\infty}^{y} \sinh [\alpha(y-\zeta)] \mathrm{Ai}\left[\mathrm{e}^{\mathrm{i} \pi / 6}(\alpha R)^{1 / 3}(\zeta-c-\mathrm{i} \alpha / R)\right] \mathrm{d} \zeta, \\
\chi_{4}(y)=\frac{1}{\alpha} \int_{-\infty}^{y} \sinh [\alpha(y-\zeta)] \mathrm{Ai}\left[\mathrm{e}^{5 \mathrm{i} \pi / 6}(\alpha R)^{1 / 3}(\zeta-c-\mathrm{i} \alpha / R)\right] \mathrm{d} \zeta .
\end{gathered}
$$

A disadvantage of using this solution is that instead of $F(z)$ in (4.9) the dispersion equation will contain a function of two parameters: $z$ and $\alpha$.

Note that the approximate solution (4.6) is the lowest-order term in an expansion of (4.11) with respect to $\alpha$. Thus, another possibility to improve on (4.6) is to take into account higher-order terms of this expansion. Also the solution (4.7) can be improved by adding higher order terms (see Lin 1955). We have found, however, that the problem of the breakdown of the approximations at a wavenumber around 1 is not resolved. The small parameter of the expansion is $\alpha /(\alpha R)^{1 / 3} \ll 1$, but because $\operatorname{Im}(c)$ is of the order $1 /(\alpha R)^{1 / 3}$ the expansion is valid for $\alpha \ll 1$ only.

A third possibility is a matched asymptotic expansion as used by Hooper \& Boyd (1987). Their solution is not uniformly valid if $\operatorname{Re}(c+1)$ is very small. However, for large enough wavenumbers this is not the case (Miles 1960, equation (5.1)) so that the results of an analysis using the stream function as given by Hooper \& Boyd can be used for $\alpha>0.1$, while Miles's results can be used for $\alpha \ll 1$. Using a matched asymptotic expansion yields results similar to the analysis of Miles and no agreement with the result of Smith \& Davis (1982), for a similar reason as discussed above. Such an analysis, however, does give a simple estimate of the growth rate and an asymptote of the neutral curvet:

$$
\left.\begin{array}{l}
\left.R(\alpha)=8 \alpha^{3}\left(1+c_{0}\right)(1+\alpha S \tanh \alpha) / c_{0}^{2}\right)^{2}\left[\tanh \alpha /\left(\alpha c_{0}\right)\right]^{2} \cosh ^{4} \alpha \\
c_{0}=-\left[1+\left(1+4 \alpha^{3} S \operatorname{coth} \alpha\right)^{1 / 2}\right] /(2 \alpha \operatorname{coth} \alpha)
\end{array}\right\}
$$

$\dagger$ The Mathematica (Wolfram 1991) programme to solve (4.9) is available from the authors or the JFM Editorial Office.

$\ddagger$ A copy of the calculation is available from the authors or the JFM Editorial Office. 

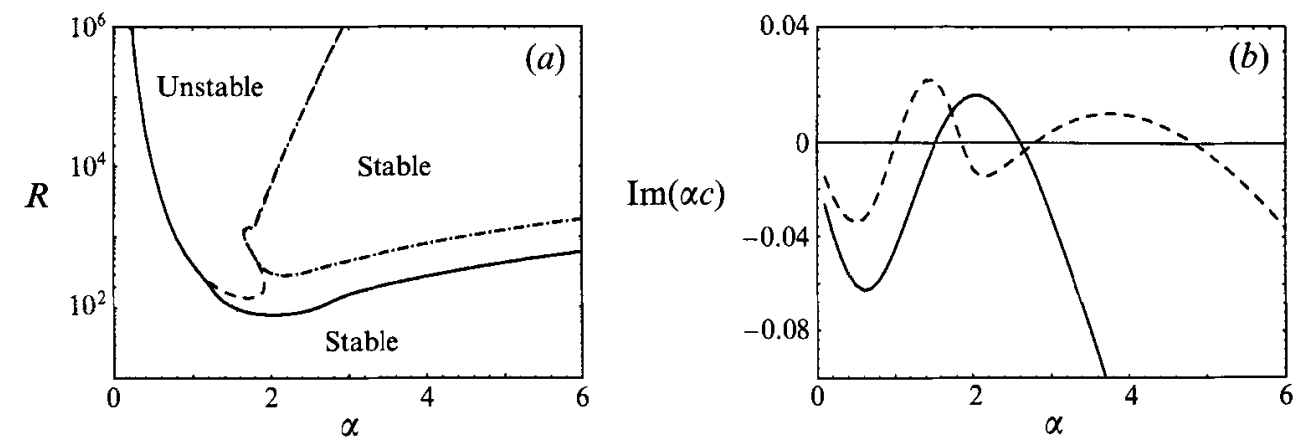

FIGURE 2. The free surface approximation. (a) Curves of neutral stability as found by Miles (1960) (dashed line), Smith \& Davis (1982) (solid) and in this paper (dashed-dotted line). (b) The growth rate $\operatorname{Im}(\alpha c)$ as a function of the wavenumber for $S=0$, for $R=100$ (solid line) and $R=400$ (dashed line).

As the asymptotic methods in this section do not improve on the results of Miles, we will use a linear combination of the exact solutions defined in (4.5), (4.11) and (4.12). We then proceed as in $\$ 4.1$ : substituting the linear combination into the boundary conditions (4.3)-(4.4), giving (4.8). To obtain that equation $(\alpha R)^{1 / 3} \gg 1$ is used, so that $\chi_{3}$ and $\chi_{4}$ are exponentially small at the interface and the wall, respectively. Because of the highly oscillatory nature of the Airy function this assumption is essential to get a dispersion equation that can be solved numerically. The expansion of the determinant gives again the dispersion equation (A 1), but with $F(z)$ now defined by (A 2) and (4.11). The expression for $\varpi_{4 i}$ is in this case found from (4.4) and (4.12). The dispersion equation thus obtained is then solved using a small Mathematica programmet.

\subsection{Resolution of the differences in neutral curves}

The neutral curve found with this programme (figure $2 a$, solid line) is the same as curve $(b)$ in figure 2 of Smith \& Davis (1982), giving a critical Reynolds number of 78.4. If the growth rate is plotted as a function of the wavenumber for $S=0$ and $R=100$ (figure $2 b$, solid line) the two points at which the growth rate is zero ( $\alpha \approx 1.5$ and $\alpha \approx 2.6$ ) correspond to the intersection of the line $R=100$ and the curve of neutral stability (solid line) in figure $2(a)$. For higher Reynolds numbers, however, there are two extra points where the growth rate is zero (figure $2 b$, dashed line). Obviously, these points form another curve of neutral stability (figure $2 a$, dasheddotted line), which has not been noted previously. This shape of the neutral curve implies that for large Reynolds numbers there is in fact a 'short wave instability' and an instability at moderate or long wavelengths. Note that the asymptotes of the neutral curve given by $R_{n}=27.05(1-3 S)^{-3} \alpha^{-7}$ (equation (6.8) of Miles 1960) and equation (4.13), correspond to the moderate wavelength instability.

If the surface tension coefficient $S$ is only slightly larger than zero, the short wave instability is stabilized by surface tension and the two branches of the neutral curve are connected again. The neutral curve is similar in shape to the curves found by Miles (see figure $2 a$, dashed line). If the surface tension coefficient is increased further the Reynolds number for which there is instability also increases and the results resemble more and more the asymptotic result found from equation (4.9). The

$\dagger$ The programme is available from the authors or the JFM Editorial Office. 
Reynolds numbers for which this is the case are, however, very large so that the flow in the liquid layer will be far from laminar.

In case of instability the most unstable mode has a wavenumber typically between 1 and 2, i.e. 3 to 6 times the thickness of the liquid layer. With increasing Reynolds number the phase velocity decreases from half the interface velocity to almost zero (in the laboratory frame). A typical dimensionless growth rate is order 0.01 .

\section{The dynamic effect of the gas}

So far we have neglected the effect of the perturbations of the flow in the gas on the stability of the liquid film and we have considered the influence of the gas through the shear of the basic flow in the liquid only. The conditions for this approximation were given by Miles (1960) and in $\S 3$. In this section the dynamic effect of the gas will be considered, as well as the validity of these conditions, by solving the system of equations given by (3.2)-(3.4), (3.6)-(3.15). These equations will be solved numerically and asymptotically neglecting the curvature of the velocity profiles, i.e. for the primary flow given by (2.5) with $A=0$ and (2.7).

\subsection{Asymptotic solution}

The solution of equation (3.4) for the region where the gas has a constant velocity, together with condition (3.7), is given by

$$
\psi(y)=C \exp [\alpha(b-y)]+D \exp [-\gamma(y-b)],
$$

where $\gamma$ is given by

$$
\gamma^{2}=\mathrm{i} \alpha \operatorname{Rrm}(m b-c)+\alpha^{2}, \quad \operatorname{Re}(\gamma)>0,
$$

and $\operatorname{Re}()$ stands for taking the real part.

An approximate solution of (3.3) that satisfies (3.12)-(3.15) is given by†:

$$
\begin{gathered}
\phi(y)=d_{1} \phi_{1}(y)+d_{2} \phi_{2}(y), \\
\phi_{1}(y)=\exp [\alpha(b-y)]-\frac{\sinh [\alpha(b-y)]}{\alpha(b-c / m)}, \\
\phi_{2}(y)=\int_{\infty}^{y}(y-\zeta) \operatorname{Ai}\left[\mathrm{e}^{\mathrm{i} \pi / 6}\left(\alpha R r m^{2}\right)^{1 / 3}(\zeta-c / m)\right] \mathrm{d} \zeta .
\end{gathered}
$$

A more accurate solution for the viscous part of $\phi(y)$ is given by (cf. (3.2) and its solution):

$$
\phi_{2}(y)=\frac{1}{\alpha} \int_{\infty}^{y} \sinh [\alpha(y-\zeta)] \mathrm{Ai}\left[\mathrm{e}^{\mathrm{i} \pi / 6}\left(\alpha R m^{2} r\right)^{1 / 3}\left(\zeta-c / m-\mathrm{i} \alpha / R m^{2} r\right)\right] \mathrm{d} \zeta .
$$

In the following we will use this solution for $\phi_{2}(y)$, because we have shown in $\S 4$ that this accuracy is required to correctly determine the imaginary part of $c$ and the neutral curves. Note that in constructing the solution it has been assumed that the viscous part $\phi_{2}(y)$, which is associated with the viscous boundary layer near the

$\dagger$ Yih (1990) constructs this solution by first approximating the conditions (3.12)-(3.15) at the virtual interface $y=b$ by two conditions in which only the 'inviscid' part of $\phi$ occurs. The same solution can also be obtained in a more formal way by solving equation (3.3) with a matched asymptotic expansion (Hooper $\&$ Boyd 1987), correct to $O(\alpha R)^{-1 / 2}$, which satisfies the four conditions at the virtual interface. 
interface, is small at the virtual interface $y=b$, i.e. $\left(\alpha R m^{2} r\right)^{1 / 3} b \gg 1$. This is a similar approximation as made in the liquid (§4), for which it was required that $(\alpha R)^{1 / 3} \gg 1$.

We then proceed as in $\S 4.1$ : the solution (5.3) for $\phi(y)$ and the solution for $\chi(y)$, given by a linear combination of (4.5), (4.11) and (4.12), are substituted into (3.6) and (3.8)-(3.11). The coefficient determinant of the resulting six equations must be zero to have non-trivial solutions:

$$
\left|\begin{array}{cccccc}
1 & 0 & \chi_{3}(-1) & 0 & 0 & 0 \\
0 & \alpha & \chi_{3}^{\prime}(-1) & 0 & 0 & 0 \\
\varpi_{1} & \varpi_{2} & 0 & \varpi_{4} & \Omega_{1} & \Omega_{2} \\
2 \alpha^{2} \chi_{1} & 2 \alpha^{2} \chi_{2} & 0 & \chi_{4}^{\prime \prime}+\alpha^{2} \chi_{4} & -\left(\phi_{1}^{\prime \prime}+\alpha^{2} \phi_{1}\right) / m & -\left(\phi_{2}^{\prime \prime}+\alpha^{2} \phi_{2}\right) / m \\
\chi_{1} & \chi_{2} & 0 & \chi_{4} & -\phi_{1} & -\phi_{2} \\
-\chi_{1}^{\prime} & -\chi_{2}^{\prime} & 0 & -\chi_{4}^{\prime} & \phi_{1}^{\prime}-\phi_{1}(1-m) / c & \phi_{2}^{\prime}-\phi_{2}(1-m) / c
\end{array}\right|=0
$$

where

$$
\begin{aligned}
& \varpi_{i}=\left.\left[c \chi_{i}^{\prime}+\chi_{i}-\frac{\mathrm{i}}{\alpha R}\left(\chi_{i}^{\prime \prime \prime}-3 \alpha^{2} \chi_{i}^{\prime}\right)-\alpha^{2} S \chi_{i} / c\right]\right|_{y=0}, \\
& \Omega_{i}=\left.\left[-r\left(c \phi_{i}^{\prime}+m \phi_{i}\right)+\frac{\mathrm{i}}{m \alpha R}\left(\phi_{i}^{\prime \prime \prime}-3 \alpha^{2} \phi_{i}^{\prime}\right)\right]\right|_{y=0} .
\end{aligned}
$$

Note that the upper-left $4 \times 4$ submatrix is the same as equation (4.8). Equation (5.7) is solved using a Mathematica programme that is similar to the one used in $\S 4.2$. This gives the complex phase velocity as a function of the wavenumber and the parameters of the problem:

$$
c=c(\alpha, R, S, m, r, b),
$$

where $b$ is calculated from $\tau=\frac{1}{2} c_{f} \rho_{g} U_{g}^{2}$ and (2.8) and is a reflection of the primary flow of the gas. Note that it has been assumed that $(\alpha R)^{1 / 3} \gg 1$ and that $\left(\alpha R m^{2} r\right)^{1 / 3} b \gg 1$. Comparing with the results in $\S 5.2$ shows that correct results are obtained if $(\alpha R)^{1 / 3}>4$ and $\left(\alpha R m^{2} r\right)^{1 / 3} b>4$.

To assess if the dynamic effect of the gas on the hydrodynamic stability of the liquid film is significant, the growth rate for realistic values of the parameters $R, S, A, m, r$, and $b$ is compared with the result for the same $R, S$, and $A$ in the free surface approximation of $\S 4$. We remark that taking the gas into account leads to an extra mode of instability, (see e.g. Miles 1962; Blennerhassett 1980; Yiantsios \& Higgins 1988 ) which will be considered in \$6. Consider a typical air/water system, so that $\rho_{l}=997 \mathrm{~kg} \mathrm{~m}^{-3}, \mu_{l}=1.0110^{-3} \mathrm{Pas}, \rho_{g}=1.21 \mathrm{~kg} \mathrm{~m}^{-3}, \mu_{g}=1.7910^{-5} \mathrm{Pas}$ and the surface tension coefficient $\sigma=0.074 \mathrm{~N} \mathrm{~m}^{-1}$. This gives $m=56.4$ and $r=0.00121$. If we take $U_{g}=40 \mathrm{~m} \mathrm{~s}^{-1}$ and $c_{f}=0.02$ (see Wallis 1969), we find from $\tau=\frac{1}{2} c_{f} \rho_{g} U_{g}^{2}$ that $\tau=19.4 \mathrm{~N} \mathrm{~m}^{-2}$. From the definition (2.3) of $U_{i}$ and $R=\rho_{l} U_{i} d / \mu_{l}$ it is found that a Reynolds number $R=400$ corresponds to $U_{i}=2.79 \mathrm{~m} \mathrm{~s}^{-1}$ and $d=0.14510^{-3} \mathrm{~m}$. The definition (3.11) of $S$ and (3.10) of $b$ then gives $S=0.0657$ and $b=0.254$. To use the asymptotic method of this section we assume that the curvature $A$ is zero. This is the case for a liquid film on a horizontal plate and approximately also for a vertical pipe ( $A$ is given by (2.4), in which the pressure gradient $P_{x}$ can often be neglected so that $A \approx 0.037$ ). In the horizontal case gravity is neglected because $F=1.83 \times 10^{-5}$. For the above values of the parameters $m, r, R, S$ and $b$, the growth rate has been calculated as a function of the wavenumber $\alpha$, with the method outlined in this section. Figure 3 shows that the dynamic effect of the gas is very large! 

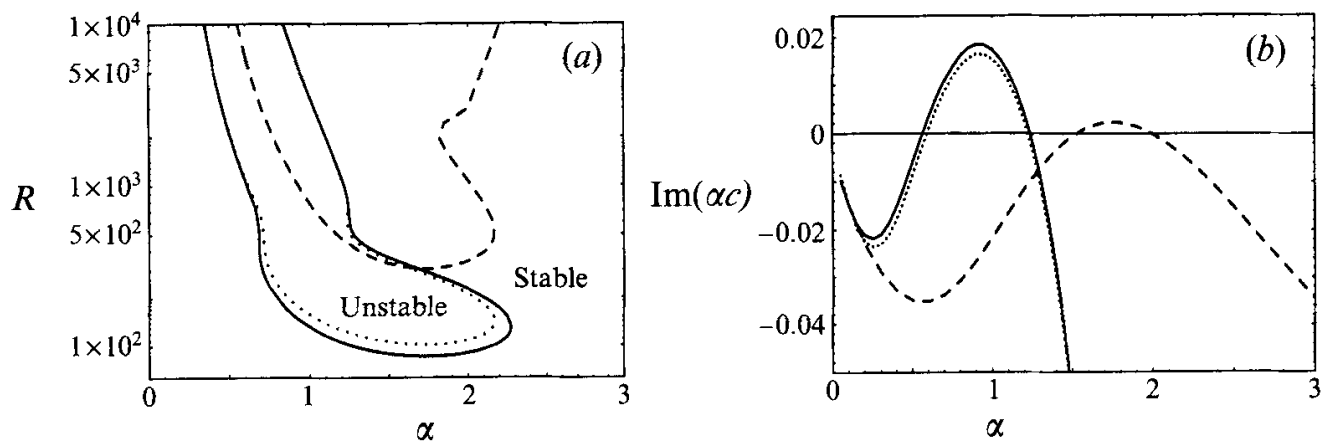

FIGURE 3. The dynamic effect of the gas on the curves of neutral stability ( $S=0.05, b=0.2)$ and the growth rate $\operatorname{Im}(\alpha c)(R=400, S=0.0657, b=0.254)$ for an air/water system. The dashed curves are for the free surface approximation considered in $\$ 4$. The solid curves have been calculated from the dispersion equation (5.7), which includes the dynamic effect of the gas in an asymptotic way. The dots have been calculated with the numerical method of $\$ 5.2$. The difference between using $F=0$ (vertical case) and $F=1.83 \times 10^{-5}$ (horizontal case) is very small.

In $\$ 3.2$ we have discussed the free surface approximation, i.e. the reduction of the interface conditions (3.8)-(3.11), to (4.4) and (4.5) if $r \rightarrow 0, m \rightarrow \infty$ and $m r \rightarrow 0$. For the air/water system considered in figure $3, r=0.00121, m=56.4$, and $m r=0.068$. Thus, the free surface approximation appears to be reasonable (Miles 1960). Figure 3, however, shows that the effect of this approximation on the growth rate, the neutral curves and the critical Reynolds number is very large. The reason for this is again that the imaginary part of the phase velocity $c$ is much smaller than the real part (cf. the explanation of the differences between the asymptotic results of Miles (1960) and the numerical results of Smith \& Davis (1982) in \$4.1). Therefore, an error of $O\left(r, m^{-1}, m r\right)$ in calculating the eigenvalue $c$ of the problem has no dramatic effect on the real part of $c$, but significantly changes its imaginary part which is often $O\left(r, m^{-1}, m r\right)$ or smaller.

Also given in figure 3 are results for the full problem calculated with the numerical method discussed next. Comparing growth rates shows that the asymptotic result is quite good, although $\left(\alpha R m^{2} r\right)^{1 / 3} b \approx 3$. The difference between the numerical solution and the asymptotic solution is even somewhat smaller if the extra term in (3.15) is taken into account (see the discussion at the end of $\S 3$ ), as can be understood from the asymptotic analysis. The agreement between the numerical and the asymptotic results is also satisfactory for the neutral curves. The asymptotic method underestimates the critical Reynolds number somewhat since $\left(\alpha R m^{2} r\right)^{1 / 3} b<2$ at the critical point.

If the dynamic effect of the gas is taken into account, the linear stability problem is characterized by three more parameters $(m, r, b)$ than in the free surface approximation, bringing the total to seven. It is then not feasible anymore to give comprehensive results, even if only neutral curves or critical Reynolds numbers are considered. Nevertheless, a few results will be given to illustrate some of the effects of the gas.

\subsection{Numerical solution}

The results of this section have been obtained with a numerical method that is based on the expansion of the eigenfunctions $\chi$ and $\phi$ in terms of Chebyshev polynomials, point collocation, and subsequent solution of the resulting generalized matrix eigenvalue problem with the QZ-algorithm (see e.g. Molar \& Stewart 1973). 
The method is similar to that used by Orszag (1971) to investigate the stability of plane Poiseuille flow and has been used by us before (see Miesen et al. 1992). Note that the interface conditions can be written in a form linear in $c$. It is therefore not necessary to solve the problem iteratively as Valenzuela (1976) does. Furthermore, the introduction of the 'virtual interface' reduces the problem that Valenzuela encountered with the choice of the size of the computing domain in the gas. If assuming a linearconstant velocity profile is undesirable, it can be efficient to introduce a second 'virtual interface' between the parts of the gas flow with steep and less steep velocity profile.

As mentioned, seven parameters characterize the problem: $\alpha, R, S, A, m, r$, and $b$. We choose the critical Reynolds number $R_{c}$ as a function of the surface tension parameter $S$, as a global indicator of the stability problem. In an experiment $m$ and $r$ will not vary, and we choose their values for an air/water system (at atmospheric pressure and $15^{\circ} \mathrm{C}$ ). Then only $A$ and $b$ are left as parameters.

If, as in the case of a horizontal plate, $A=0$ we need only consider the effect of different values of $b$ on $R_{c}(S)$. In an experiment for constant liquid flux, however, $S$ depends on the gas speed $U_{\mathrm{g}}\left(S=\sigma /\left[\rho_{l} U_{i}\left(U_{\mathrm{g}}\right)^{2} d\left(U_{\mathrm{g}}\right)\right]\right)$; we therefore consider the dependence of $b$ on $U_{g}$. From $\tau=\frac{1}{2} c_{f} \rho_{g} U_{g}^{2}$ we see that $\tau \propto U_{g}^{2}$; from the definitions of $R$ and $U_{i}$ (equation (2.3)) we find that $d \propto U_{\mathrm{g}}^{-1}$ and $U_{i} \propto U_{\mathrm{g}}$, because $R$ is constant for constant liquid flux and a linear velocity profile. Thus $b=U_{g} /\left(m U_{i}\right)$ is constant with varying gas velocity and $S \propto U_{g}^{-1}$. It is therefore useful to consider $R_{c}(S)$ at constant values of $b$. If $A \neq 0$ things are more complicated, because in that case $R$, $S$, and $b$ are less simple functions of $U_{g}$.

Including the dynamic influence of the gas can lead to more complicated neutral curves, which leads to apparently strange discontinuities in the function $R_{c}(S)$ (figure 4). The neutral curve for $S=0.05$ (solid line, figure $4 a$ ) is the same as the dotted curve in figure 3(a). If the surface tension coefficient is increased above approximately $S=0.082$, the curve splits and an 'island of instability' is formed. If $S$ is increased above the value for which the island disappears $(S \approx 0.092)$, the critical Reynolds number jumps to the minimum of the other neutral curve, showing as a discontinuity in $R_{c}(S)$ (figure $4 b$ ). Blennerhassett (1980) also found such 'islands of instability' (for certain gas velocity profiles), but for the 'interfacial mode' which is discussed in the next section.

From figure $4(b)$ it is again clear that the dynamic influence of the gas can be appreciable, and can lead to both a larger and a smaller critical Reynolds number. Compared to the free surface approximation the dependence of the critical Reynolds number on the inverse Weber number $S$ is less strong, especially for smaller values of $S$ or for larger values of $b$. Also note that the critical Reynolds number can (slightly) decrease if $S$ increases and can strongly depend on the value of $b$. This is because the maximum growth rate is attained for $\alpha \approx 1$, because for very small or very large wavenumbers the growth rate will hardly change if $b$ varies, since the wave is either much shorter or much longer than the thickness of the sublayer. If $b$ is smaller than, say 0.2 the extra term in (3.15) becomes important, which means that the approximation of the gas velocity profile by a linear-constant profile is not correct anymore. For example, the minimum in the curve for $b=0.1$ in figure $4(b)$ goes from 78 to 53 if the extra term is included.

In figure $5(a)$ the influence of the profile curvature is investigated. It is relatively weak for $S$ below any discontinuity and very large above it (if $A$ is large enough). Finally, in figure $5(b)$ it is shown that the viscosity and density ratio of the gas and 

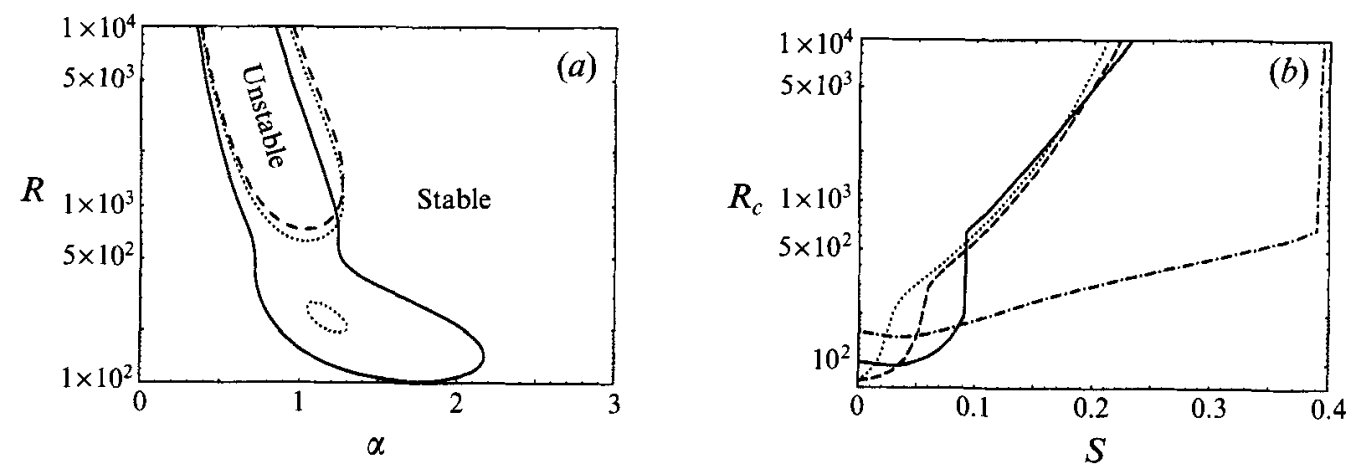

Figure 4. Neutral curves for $b=0.2(m=56.4, r=0.00121, A=0, F=0)$ and $S=0.05$ (solid line), $S=0.09$ (dotted), $S=0.10$ (dashed). (b) The critical Reynolds number as a function of the surface tension coefficient $S$ for $b=0.1$ (dashed), $b=0.2$ (solid), and $b=0.5$ (dashed/dotted). The dotted line is for the free surface approximation.
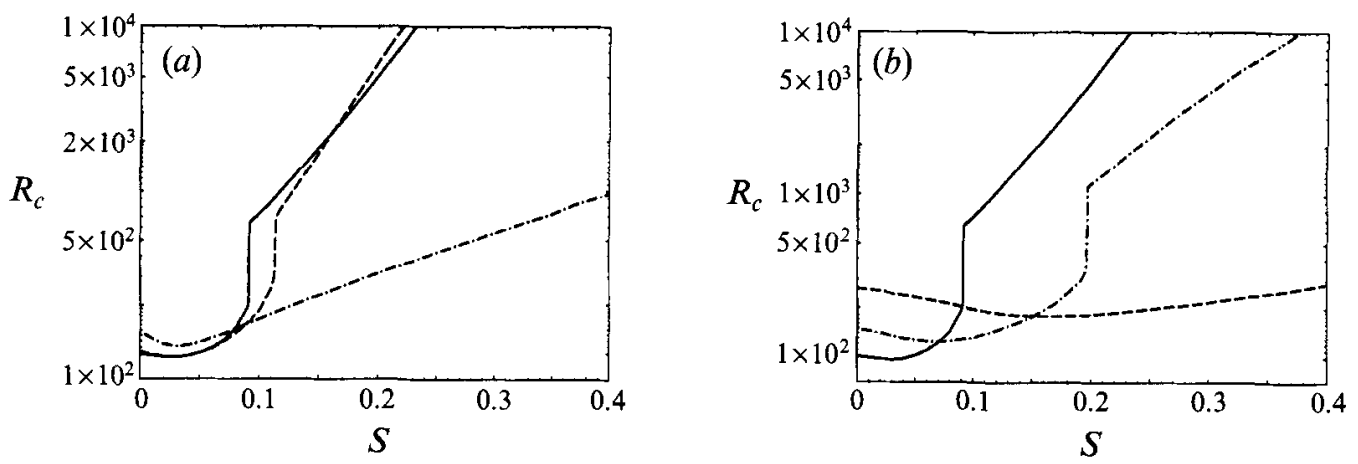

FIGURE 5. The critical Reynolds number as a function of the surface tension coefficient $S$. (a) $b=0.2, m=56.4, r=0.00121$ and $A=0$ (solid line; also given in figure 4), $A=0.1$ (dashed), $A=0.4$ (dashed/dotted); $(b)$ is for $b=0.2, A=0$ and $m=56.4, r=0.00121$ (solid line; also given in figure 4 and $a$ ), $m=112.8, r=0.00121$ (dashed), $m=56.4, r=0.00242$ (dashed/dotted).

the liquid have a marked effect on the curve $R_{c}(S)$. A somewhat more viscous liquid can lead to a critical Reynolds number that is either an order of magnitude smaller, or several times larger than the critical Reynolds number of an air/water system!

An estimate of the dynamic effects of the gas has also been given by Miles (1960). Following the analysis by Feldman (1957), Miles' result, equation (8.5), is obtained using interface conditions that lack the first-order corrections for the displacement of the interface. If we compare the growth rates, as found in the free surface approximation and as found from Miles' equation (8.5), the difference is small. We have seen, however, that the dynamic effects of the gas are large. Evidently, using the more exact form of the interface conditions is essential and this is a major flaw in the paper by Feldman (1957), as was noted previously by Blennerhassett (1980) and Hooper \& Boyd (1983).

From the results presented in this section we conclude that in order to describe waves with a phase velocity smaller than the maximum liquid velocity in the film correctly, it is necessary to take the dynamic effect of the gas into account, i.e. the free surface approximation in the description of these waves is incorrect. 


\section{The interfacial mode}

Until now we have considered the mode which is present whether or not the gas is included in the description of the sheared liquid layer. Since this mode (the internal mode) has a phase velocity smaller than the maximum liquid velocity, the critical layer is in the liquid. By including the dynamics of the gas flow a second mode is introduced with a phase velocity that is, in general, larger than the maximum liquid velocity but much smaller than the gas velocity. Thus the critical layer is in the viscous sublayer. Hence this mode is called the 'interfacial mode' and velocity profile curvature can be neglected. Note, however, that if the liquid is deep and gas speeds are relatively low, the critical layer shifts out of the viscous sublayer and velocity profile curvature becomes important (Miles 1957).

\subsection{Results of van Gastel}

The interfacial mode in fact corresponds to what are called capillary-gravity waves, which are studied in the context of the generation of surface waves by wind (see e.g. Miles 1962a). If the thickness of the fluid layer $d$ is chosen larger than a few times the length of the disturbance, growth rates are found that are the same as for capillary waves on deep water. The easiest way to show this is by reproducing the result given in figure 5 of the paper by van Gastel, Janssen \& Komen (1985) for the growth rate of capillary waves generated on deep water by wind with a linear velocity profile; the (infinite) linear wind profile is simulated by choosing a large enough value of the boundary layer thickness $b$ in our description, so that results become independent of this parametert.

We, however, find an approximately 30\% larger growth rate than van Gastel et al. (1985). This improves only slightly if their different liquid velocity profile is used (in our notation, given by $\left(U_{0} / U_{i}\right)\left[\exp \left(U_{i} y / U_{0}\right)-1\right]$ with $U_{0}$ the interfacial velocity). We therefore attribute the difference to the asymptotics used in van Gastel et al. (1985) for this special case of a linear wind profile. If the results for a linear-logarithmic wind velocity profile (2.6) are compared, the difference is very small (figure 6). This small difference could be due to the asymptotic approximation used in van Gastel et al. (1985) and the fact that we have used a finite number of polynomials and a finite thickness for both the liquid and the gas.

We have now shown that the mode that enters the problem if the dynamics of the gas flow is included into the description is akin to capillary waves. For waves that are not too long, this mode has a positive phase velocity relative to the gas/liquid interface and its magnitude is of the order of the liquid velocity. Reproducing the results of van Gastel et al. (1985) has also been a second check, after comparing with the asymptotic results in $\$ 5.1$, that the code for the numerical solution of the problem works well. A third check (not given here) was a comparison with the asymptotic results of Yih (1990) for the linear stability of a sheared film of a very viscous liquid.

For certain parameter values a third unstable mode was found. This mode is associated with the virtual interface and is therefore not physically relevant: the corresponding eigenvalue disappears if $c$ is neglected in the conditions (3.12)-(3.15).

$\dagger$ The parameters are calculated as follows. For given friction velocity $u_{*}$ the shear force is given by definition as $\tau=\rho_{\mathrm{g}} u_{\text {. }}^{2}$. Then, the thickness of the liquid layer is chosen to be a few times the wavelength. This gives the velocity $U_{i}=d \tau / \mu_{l}$, the Reynolds number $R=\rho_{l} U_{i} d / \mu_{l}$, and the surface tension parameter $S=\sigma / \rho_{l} U_{i}^{2} d$. For $b$, a value of 1 is large enough, in general. The dimensional growth rate of van Gastel et al. (1985) is given by $2 \alpha \operatorname{Im}(c) U_{i} / d$. 


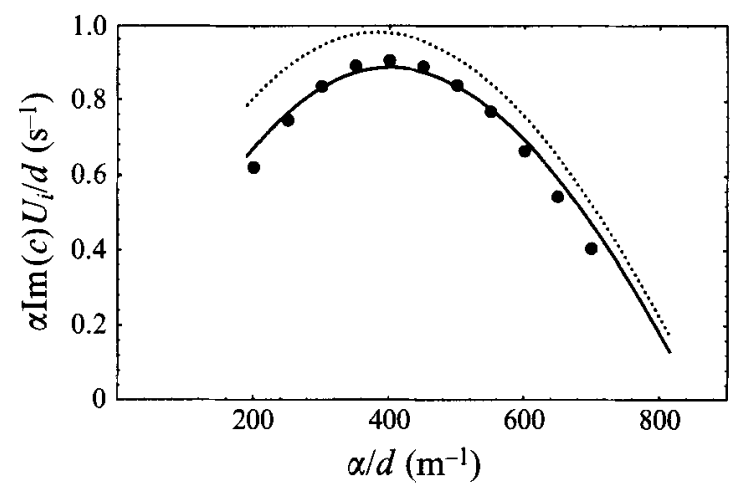

FIGURE 6. The dimensional growth rate $\alpha \operatorname{Im}(c) U_{i} / d$ of the interfacial mode as a function of the dimensional wavenumber $\alpha / d$, for a linear-logarithmic velocity profile in the gas, $m=55.6, r=0.0012, R=1.9810^{5}, S=1.1110^{-4}, F=5.4010^{-2}, A=0, b=1$, corresponding to (in SI units) $g=9.8, \sigma=7.25 \times 10^{-2}, \rho_{l}=1000, \rho_{g}=1.2, \mu_{l}=0.001, \mu_{g}=1.810^{-5}, d=0.06, U_{i}=3.30$ and $u_{*}=\left(\tau / \rho_{\mathrm{g}}\right)^{1 / 2}=0.214 \mathrm{~m} \mathrm{~s}^{-1}$. The interfacial velocity $U_{0}=0.098 \mathrm{~m} \mathrm{~s}^{-1}$ and the parameter $s$ in (2.6) is 5 . The points have been copied from figure 5 in the paper by van Gastel et al. (1985). The curves have been determined numerically, using the exponential (solid line) and linear velocity profile (dotted line) in the liquid.

\subsection{The internal and the interfacial mode}

We will now discuss the result (figure 7) of a calculation of the growth rate for both the internal and the interfacial mode, for parameter values that have also been used in figure 3. Furthermore, we will briefly consider the validity of the approximation (2.7) of the velocity profile in the gas. From figure 7 it is clear that the growth rate of the interfacial mode is an order of magnitude larger than the growth rate of the internal mode; this is the general picture. Nevertheless, if the dimensional growth rate of the internal mode is considered by multiplying by $U_{i} / d=19200 \mathrm{~s}^{-1}$, a maximum growth rate of approximately $300 \mathrm{~s}^{-1}$ is found which is large enough for this mode to be observed. Furthermore, we should keep in mind that we have considered wave growth in the small-amplitude limit and wave growth will saturate as finite-amplitude effects become important. In interpreting experiments, the internal mode should therefore not be neglected a priori. Some experimental evidence that the internal mode is indeed important will be discussed in the next section.

Comparing the results of a calculation using the velocity profiles (2.6) and (2.7) gives us an idea of whether the simplification of the velocity profile in the gas is justified. In both cases a linear velocity profile in the liquid is used. For the calculation with the logarithmic profile (2.6), the virtual interface, beyond which the gas velocity is assumed constant, is chosen to be far enough from the liquid/gas interface for the eigenvalues not to change substantially anymore. In figure 7 it can be seen that the differences between the results for the two velocity profiles are reasonably small and are smallest for high wavenumbers, i.e. waves that are affected by the linear sublayer only. For small wave numbers the differences are small if the maximum velocities of the two profiles are equal, because very long waves are not changed by the details of the flow at the interface. The largest difference between the growth rates found with the two velocity profiles is for $\alpha=O(1)$. We conclude that the approximation (2.7), which allows for an asymptotic solution of the problem (\$5.1) or for a more effective numerical solution (less polynomials), is valid unless a high accuracy is required or 


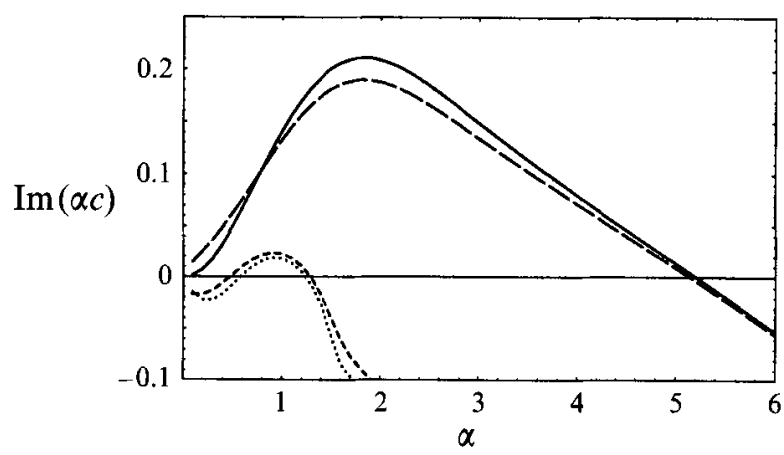

FIGURE 7. The dimensionless growth rate of the interfacial and the internal modes as a function of $\alpha$ for the velocity profile (2.7) in the gas (solid and dotted line respectively). The short-dashed and long-dashed lines correspond to the liquid and the gas mode, respectively, for the linear-logarithmic profile (2.6) with $b \approx 3.5, s=5$ and $u_{*}=\left(\tau / \rho_{g}\right)^{1 / 2}=4.00$.

unless the critical layer is not well within the viscous sublayer so that velocity profile curvature is important.

\section{Discussion}

In the foregoing we have considered the theoretical problem of the stability of a liquid film that is sheared by a gas. It has been shown that using the free surface approximation leads to quite different results for, for example, the critical Reynolds number. Including the dynamics of the gas in the problem leads to a second mode of instability, corresponding to capillary waves. In this section we will compare predictions of the (in)stability of sheared liquid films with experimental and theoretical results as reported in papers by Cohen \& Hanratty (1965) and Craik (1966). This also offers the opportunity to compare the two methods to predict the instability of a sheared liquid: using coupled Orr-Sommerfeld equations and using the 'divided attack' (see Benjamin 1959). To our knowledge this has not been done before. In a comparison between experiment and theory the second method is mostly used, in which the Orr-Sommerfeld equation for the liquid is solved considering the waveinduced stress variations in the gas as given. On the one hand this leads to much simpler equations to be solved, but on the other hand the problem is shifted to finding correct expressions for the stresses.

\subsection{Cohen \& Hanratty}

Cohen \& Hanratty (1965) investigate the conditions for the onset of instability of liquid films that are several millimetres thick, having a viscosity between one and ten times that of water. The experiments are done in a horizontal, long, wide channel approximately $1 \mathrm{in}$. high. For a fixed flow rate in the film, i.e. for a constant liquid Reynolds number, they determine the gas velocity (and film thickness) at which the first waves develop. This critical gas velocity is compared with predictions from a theoretical analysis in which the Orr-Sommerfeld equation for the liquid is approximately solved. The wave-induced stresses in the gas at the liquid/gas interface are calculated using a method described by Miles $(1962 b)$. It is assumed that the gas is of infinite extent - an assumption we will also make - which is correct if $2 \pi H / \lambda \gg 1$, where $H$ is the height of the channel and $\lambda$ is the wavelength. This 


$\begin{array}{ccccccccc}\text { No. } & \text { Fluid } & R & \mu_{l}(\mathrm{mPas}) & \rho_{l} & \mu_{\mathrm{g}}(\mu \mathrm{Pa} \mathrm{s}) & \rho_{\mathrm{g}} & d(\mathrm{~mm}) & R_{\mathrm{g}} \\ 1 & \mathrm{H}_{2} \mathrm{O} & 176 & 0.929 & 1000 & 18.3 & 1.14 & 1.89 & 4050 \\ 2 & \mathrm{H}_{2} \mathrm{O} & 320 & 0.933 & 1000 & 18.3 & 1.14 & 3.54 & 2760 \\ 3 & \mathrm{H}_{2} \mathrm{O} & 494 & 0.925 & 1000 & 18.3 & 1.14 & 4.91 & 1870 \\ 4 & \mathrm{G}-\mathrm{I} & 280 & 3.95 & 1110 & 18.5 & 1.11 & 6.41 & 2330 \\ 5 & \mathrm{G}-\mathrm{I} & 122 & 3.89 & 1110 & 18.5 & 1.11 & 4.48 & 3600 \\ 6 & \mathrm{G}-\mathrm{II} & 96 & 11.2 & 1170 & 18.5 & 1.16 & 7.32 & 3650 \\ 7 & \text { G-II } & 42 & 11.2 & 1170 & 18.5 & 1.16 & 5.28 & 4620\end{array}$

TABLE 2. Experimental conditions and results from Cohen \& Hanratty (1965). The liquid Reynolds number $R$ is based on the maximum liquid velocity $U_{i}$ and is twice the Reynolds number $N_{R}$ in Cohen \& Hanratty (1965). The gas Reynolds number $R_{g}$ is based on the gas properties, the average gas velocity and the height of the channel $(H=0.0258 \mathrm{~m})$ minus the film thickness.

condition is barely satisfied for the wavelengths in the experiments $(2 \pi H / \lambda \approx 5)$, but it is expected that this is good enough.

In table 2 the conditions for the experiments given in table 2 of Cohen \& Hanratty and the results for the critical Reynolds number of the gas are given in SI units. The results of the calculations by Cohen \& Hanratty for the critical gas Reynolds number are given in the second column of table 3. The agreement between the experimental value of the critical Reynolds number and the theoretical one is quite good, although the theoretical value is systematically higher (on average $23 \%$ ).

With the method presented in this paper the critical gas velocity can be obtained as follows. If we use the model with the linear-constant gas velocity profile the only parameter that can be varied is $b$, which is proportional to the maximum gas velocity (equation (2.8)). If a value of $b$ is chosen, $U_{i}, S$ and $F$ can be calculated because $d$ and $R$ are known from the experiment. Thus $b$ is varied to determine for which value the maximum growth rate is zero, giving the critical maximum gas velocity $U_{g m}=(1+m b) U_{i}$ (in the laboratory frame). The average gas velocity is (after some simple algebra) given by $U_{g a}=U_{i}\left[1+m b-m b^{2} d /(H-d)\right]$. The mode that is most unstable has a phase velocity that is typically three times the maximum liquid velocity and is thus the interfacial mode. The results for this case are given in table 3 (third and fourth column). The agreement with the experiment is better than obtained by Cohen \& Hanratty (1965), some critical gas Reynolds numbers being too high, others being too low and with an average error of $14 \%$.

Better agreement may be expected if a gas velocity profile as given by equation (2.6) is used (the virtual interface $y=b$ is then assumed to be halfway into the gas layer). The problem in this case is that all parameters are fixed if $R$ and $d$ are given. $U_{i}$ is fixed, so that $\tau$ is fixed through the definition of $U_{i}$ (equation (2.3)) and this completely determines the velocity profile in the gas. This problem can be solved in several ways. Cohen \& Hanratty (1965) solve the problem by using gas velocity profiles measured in the dry channel and calculating for which profile and corresponding average gas velocity the film is neutrally stable. For our calculations this corresponds to using the measured $R$ and $d$ for the liquid film and varying $\tau$ in the gas velocity profile (2.6). The shear stress in the basic flow is then, however, discontinuous at the interface. Throughout this report we have seen that such an approximation of the interface conditions can lead to a considerable error in the growth rate and thus in the critical Reynolds number. This is confirmed by the results for the critical gas Reynolds number, especially for the experiments with the more viscous fluids (table 3 ; the 


\begin{tabular}{|c|c|c|c|c|c|c|c|c|c|c|}
\hline \multirow[t]{2}{*}{ No. } & \multirow{2}{*}{$\begin{array}{c}\text { Cohen } \\
R_{\mathrm{g}}\end{array}$} & \multicolumn{2}{|c|}{ Linear-constant } & \multicolumn{2}{|c|}{ Discontinuous stress } & \multicolumn{2}{|c|}{ Fixed $d$} & \multicolumn{2}{|c|}{ Fixed $R$} & \multirow{2}{*}{$\begin{array}{c}\text { Exp. } \\
R_{g}\end{array}$} \\
\hline & & $\bar{b}$ & $R_{g}$ & $u$. & $\overline{R_{g}}$ & $u *$ & $R_{\mathrm{g}}$ & $u$. & $R_{g}$ & \\
\hline 1 & 4500 & & 0 & & 4200 & 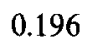 & 39 & 0.1 & 4020 & 4050 \\
\hline 2 & 310 & & & & & & & & 3050 & \\
\hline 3 & 2500 & & 16 & 0.1 & 0 & 0.1 & 21 & 0.1 & 2280 & $18^{\circ}$ \\
\hline 4 & 3300 & 0.06 & 2520 & 0.0 & 13 & 0.202 & 30 & 0.1 & 2330 & 23 \\
\hline 5 & 4100 & 0.1 & 2990 & 0.1 & 2520 & 0.233 & 4080 & 0.207 & 3540 & 3600 \\
\hline 6 & 4300 & 0.0 & 4200 & 0.129 & 1830 & 0.269 & 4260 & 0.222 & 3400 & 3650 \\
\hline 7 & 5900 & 0.0795 & 4710 & 0.190 & 3230 & 0.297 & 5420 & 0.269 & 4830 & 4620 \\
\hline
\end{tabular}

TABLE 3. The critical Reynolds number for the gas calculated in several ways. In the second column the values found by Cohen \& Hanratty (1965) are given. The other values are calculated by us with the linear-constant velocity profile in the gas, with the linear-logarithmic velocity profile in the gas and $d$ and $R$ from the experiment so that the shear stress is discontinuous at the interface, with only $d$ from the experiment, and with $R$ from the experiment. The last column gives the critical Reynolds number found experimentally.

average of the gas velocity has been determined numerically from (2.6)). The reason why the agreement between the experiments and the calculations in the paper by Cohen \& Hanratty (1965) is fairly good is unclear, because the same approximation is made there. As an ad hoc explanation we could argue that the approximation made in calculating the wave-induced stresses in the gas (for which it is assumed that the gas has an interface with a solid) somehow compensates for the discontinuity in the shear stress. Two other solutions are to either consider the value of $d$ or of $R$ as unknown; then the value of $\tau$ can be adjusted to obtain neutral stability. The results for these two cases are also given in table 3 . If $d$ is fixed, the critical gas Reynolds number found is on average $14 \%$ too high. The corresponding values of the liquid Reynolds number $R$ which follow from $\tau$ and $d$ are, in general, not in very good agreement. Note, however, that in Cohen \& Hanratty (1965) they are taken to be given. From table 3 it can be seen that if $R$ is fixed, the agreement for the critical gas Reynolds number is even better. The average error in this case is only $7 \%$ (probably within the experimental accuracy) and 3.5\% if the experiment with the highest Reynolds number is omitted (the film is probably not laminar in that case). The corresponding values of the film thickness $d$, which follow from $\tau$ and $R$, are again not in good agreement. Another possibility, which we will not pursue any further here, is to use a more accurate gas velocity profile with an extra parameter. This will lead to results at least as good as by fixing $R$.

In conclusion the critical gas velocity for the generation of capillary waves on liquid films of a few millimetres thick can be predicted well with a linear stability analysis based on the coupled Orr-Sommerfeld equation for the liquid film and the gas. The error in the prediction of the critical gas speed (10\% or less) appears to be of the order of the experimental error, which is better than obtained using a divided attack.

\subsection{Craik}

Craik (1966) has done some nice experiments with an apparatus similar to that of Cohen \& Hanratty (1965), with the difference that the channel height could be either 1 or 6 in. Craik's experiments offer the possibility to check our theoretical work for films thinner than studied by Cohen \& Hanratty (1965), i.e. with a thickness between $0.1 \mathrm{~mm}$ and $1.5 \mathrm{~mm}$. Cohen \& Hanratty fix the liquid flow rate and increase the air flow until waves appear. Craik keeps the air flow fixed and decreases the liquid 
flow rate: (i) at first waves are present; (ii) for a lower liquid flow rate, i.e. for a thinner film, the film becomes stable; (iii) on decreasing the flow rate further the film becomes unstable again! The first two events can be easily understood as the waves are of the same type as observed by Cohen \& Hanratty (1965). On decreasing the film flow rate, i.e. the film Reynolds number, the critical gas Reynolds number increases (see tables 2 and 3) and when it becomes larger than the Reynolds number of the gas flow in the experiment, the film is stable. The waves in (iii) were not observed by Cohen \& Hanratty (1965) because the films on which they occur are thinner $(d<0.5 \mathrm{~mm})$ than studied there. These waves have phase velocities that are approximately equal to the maximum liquid velocity, contrary to the waves observed in (i) which move much faster. Therefore, Craik calls the waves in regime (i) 'fast waves' and the waves in regime (iii) 'slow waves'. His experiments furthermore show that if the air velocity is sufficiently large the stable regime (ii) disappears and the transition between the two wave types is continuous.

With a theoretical analysis Craik (1966) shows that the mechanism for the generation of 'slow waves' is the 'heaping up' of liquid by the component of the tangential stress that is in phase with the wave slope, and that this mechanism is dominant for sufficiently thin films. The agreement between Craik's theory and the observations for 'slow waves' is quite good. However, the transition to 'fast waves' and the thickness for which a film is most stable is not found from Craik's solution of the stability problem. We will now compare Craik's results (to which references is made by use of square brackets [ ]) to calculations along the lines described in this paper.

Craik makes the following approximations: (i) the gas is of infinite extent (the only approximation listed here we will also make); (ii) a 'divided attack'; (iii) $\alpha^{2} \ll 1, \alpha R<O(1), \alpha R|c|<O(1)$; (iv) approximate expressions for the stresses of the gas on the wavy boundary (equations [5.2]-[5.4]). Craik's result with these approximations is given by [6.5]:

$$
\alpha^{2} S+F^{2}-\Pi+\frac{3 \mathrm{i} \Sigma}{2 \alpha}=(1-c)\left[\frac{3}{\mathrm{i} \alpha R}+\frac{6}{5}(1-c)-\frac{7}{8}+\frac{27}{5} \frac{\alpha^{2}}{\mathrm{i} \alpha R}\right],
$$

where $c$ is the phase velocity in the laboratory frame, and the normal stress $\Pi$ and tangential stress $\Sigma$ are given by [5.2]-[5.4]:

$$
\left.\begin{array}{l}
\Pi=\left(\alpha / R c_{f}\right)\left[I-\sqrt{3} s+\mathrm{i}\left(2 c_{f}-s\right)\right], \quad \Sigma=\left(2 \beta I / \sqrt{3} c_{f}\right) \mathrm{e}^{\mathrm{i} \pi / 3} \alpha^{3}(\alpha R)^{-4 / 3}, \\
s=0.644 \Delta I, \quad \Delta=\left(I / c_{f}\right)\left(m^{2} r \alpha R\right)^{-2 / 3} \alpha^{2}, \quad \beta=1.732\left(m^{2} r\right)^{-1 / 3}, \\
I=\int_{0}^{H / 2 d}\left[U(y) / U_{g}\right]^{2} \mathrm{e}^{-\alpha y} \mathrm{~d}(\alpha y),
\end{array}\right\}
$$

where $U(y)$ is the dimensional gas velocity profile, with $U(0)=0$ and $U(H / 2 d)=U_{g}$; $H$ is again the channel height $\dagger$. Here we will use the velocity profile given by equation (2.7), although we have seen that the more realistic velocity profile given by equation (2.6) gives results that can be quantitatively different. Calculations can, however, be done much more efficiently for this simpler velocity profile, because considerably fewer Chebyshev polynomials are needed and the integral $I$ given by (7.2) can be found analytically.

Craik further simplifies the above expression by: (v) assuming $I$ to be constant; (vi) neglecting the component of the normal stress in phase with the wave slope and

$\dagger$ An extra factor $(m r)^{-2 / 3}$, which is missing in Craik's paper (Craik 1993, personal correspondence), has been included in the expression for $\Delta$ in equation (7.2). 


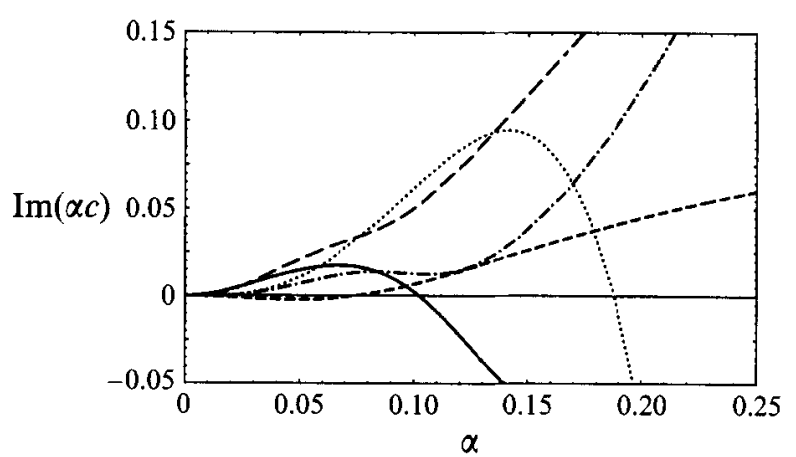

FIGURE 8. The dimensionless growth rate plotted against the wavenumber, for a water film of thickness $2 \times 10^{-4} \mathrm{~m}$ with $R=7$. The solid line is the result of the numerical solution of the coupled Orr-Sommerfeld equations, where we have used that $c_{f}=0.0015$ (see Craik 1966) so that $U_{\mathrm{g}}=13.9 \mathrm{~m} \mathrm{~s}^{-1}$. The long-dashed line is found from (7.1)-(7.2). The dotted-dashed line is found from the same expressions but with $I / c_{f}=220$. The short-dashed line is found when the approximation (7.3) is used in (7.1), and the dotted line follows from (7.4) and corresponds to figure 10 of Craik (1966).

the component of the shear stress in phase with the wave height (leading to [8.1]):

$$
\Pi=\alpha I / R c_{f}, \quad \Sigma=\mathrm{i}\left(\beta I / c_{f}\right) \alpha^{3}(\alpha R)^{-4 / 3} ;
$$

(vii) assuming that $|\operatorname{Im}(\alpha c R)| \ll 1$ so that [6.5] gives $\operatorname{Re}(c)=1$ and [7.3]:

$$
\operatorname{Im}(\alpha c R)=\frac{(\alpha R)^{2}}{3}\left(\Pi+\frac{3 \Sigma}{2 i \alpha}-\alpha^{2} S-F^{2}\right),
$$

The effect of these approximations is illustrated by figure 8, showing large differences between the successive approximations. It is remarkable, however, that the crudest approximation (7.4), which Craik uses in his discussion of the experiments, gives a point of neutral stability that is very close to that found from the original expressions (7.1) and (7.2), contrary to intermediate approximations. A comparison using neutral curves which are more important in explaining observations, is given in figure 9. Especially important is the minimum of such a curve, i.e. the critical Reynolds number $R_{c}$. The differences between the approximations are appreciable again but the critical values of the stress are reasonably close: $\tau_{c}=0.0711 \mathrm{~N} \mathrm{~m}^{-2}$ (numerical), $0.0724 \mathrm{~N} \mathrm{~m}^{-2}$ (found from (7.1) and (7.2)), $0.0895 \mathrm{~N} \mathrm{~m}^{-2}$ (expression (7.4)).

Whether or not the shear stress of the gas on the liquid film is larger than $\tau_{c}$ determines if waves are seen. We therefore consider the critical shear stress as a function of the film thickness (figures 10 and 11). It is important to note the qualitative difference between the dotted curve and the solid and dashed curves which have a maximum. This maximum is essential in understanding the sequence of observations (i)-(iii) listed at the start of this subsection. In an experiment a horizontal line is traversed in the $\left(d, \tau_{c}\right)$-plane. The starting point (i) of the experiment corresponds to an unstable film; if the film thickness is decreased sufficiently, the stable area (ii) under the curve is reached; if the film thickness is decreased further, the film becomes unstable again (iii). The maximum in the curve $\tau_{c}(d)$ is also observed experimentally. Craik notes that the film is maximally stable for $d=0.46 \mathrm{~mm}$ ( $1 \mathrm{in}$. channel); we find $d=0.35 \mathrm{~mm}$. 


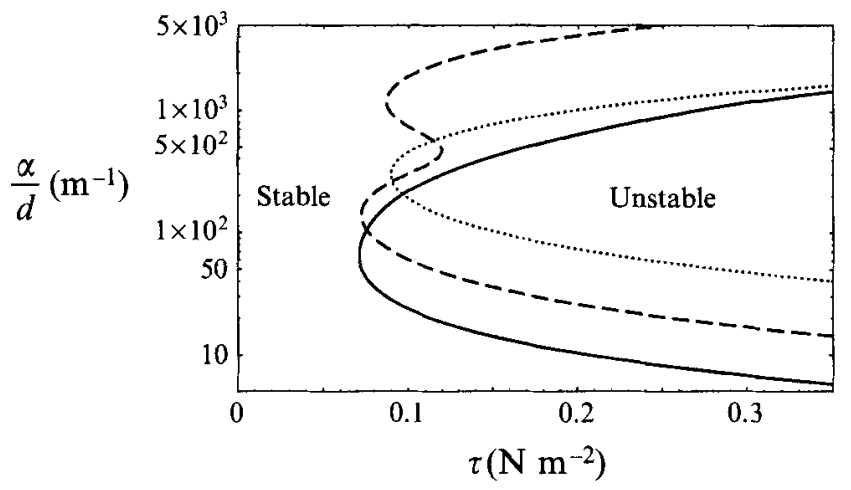

FigURE 9. Curves of neutral stability, in the $\alpha / d$ vs. $\tau=U_{i}^{2} R /\left(\rho_{l} d^{2}\right)$ plane, for a water film of thickness $0.215 \mathrm{~mm}$. The solid line has been found numerically using $c_{f}=0.0021$ (which follows from table 1 of Craik 1966). The dashed line is found from (7.1)-(7.2). The dotted line corresponds to figure 9 of Craik (1966), and follows from (7.4) with $I / c_{f}=220$.
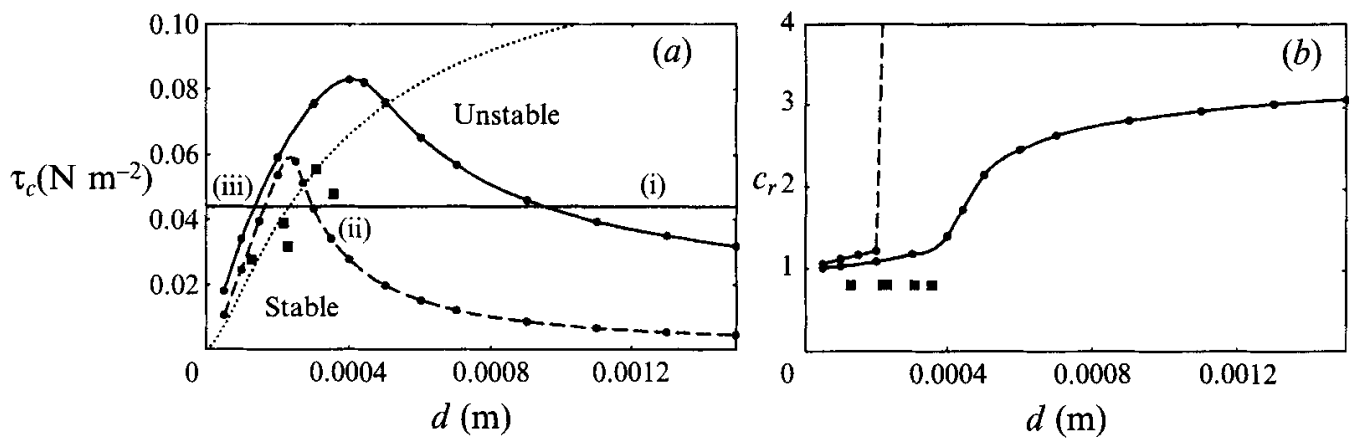

FigURE 10. A comparison between the experimental results (squares) for 'slow waves' in the 6 in. channel and theory: (a) the critical shear stress $\tau_{c}$ vs. the film thickness $d ;(b)$ the (dimensionless) phase speed at the critical point as a function of $d$ (note the transition from slow to fast waves at $d \approx 0.0004$ ). The solid lines are found numerically using $c_{f}=0.0015$ (see Craik 1966) The dashed lines are found from (7.1) and (7.2) (the dots denote the points that we have calculated). The dotted line in (a) corresponds to figure 11 of Craik (1966), and follows from (7.4) with $I=0.6\left(I / c_{f}=400\right)$. The horizontal line corresponds to a trajectory that is followed in an experiment.

The parts of the curve to the left and to the right of the maximum in $\tau_{c}(d)$ indeed correspond to 'slow' and 'fast waves', respectively, as can be seen from figures $10(b)$ and $11(b)$. For very thin films the phase speed is equal to the velocity at the surface of the film, i.e. $c=1$. If the film thickness is increased above the value for which $\tau_{c}(d)$ has its maximum the phase speed suddenly increases, as is the case in experiments. We conclude that qualitatively the agreement between the observations and theory is excellent if the numerical solution is used or if (7.1) and (7.2) are used without further approximation.

To make a more quantitative comparison the critical conditions found from tables 1 and 2 in Craik (1966) are included in figures 10 and 11, respectively. From figure 10, we see that the agreement between the experiments for 'slow waves' and the boundary between a stable and an unstable film, as found from (7.1) and (7.2) is good (although one would expect the experiments to be a bit more into the unstable area). Unfortunately, the more exact solution of the stability problem, i.e. our numerical 

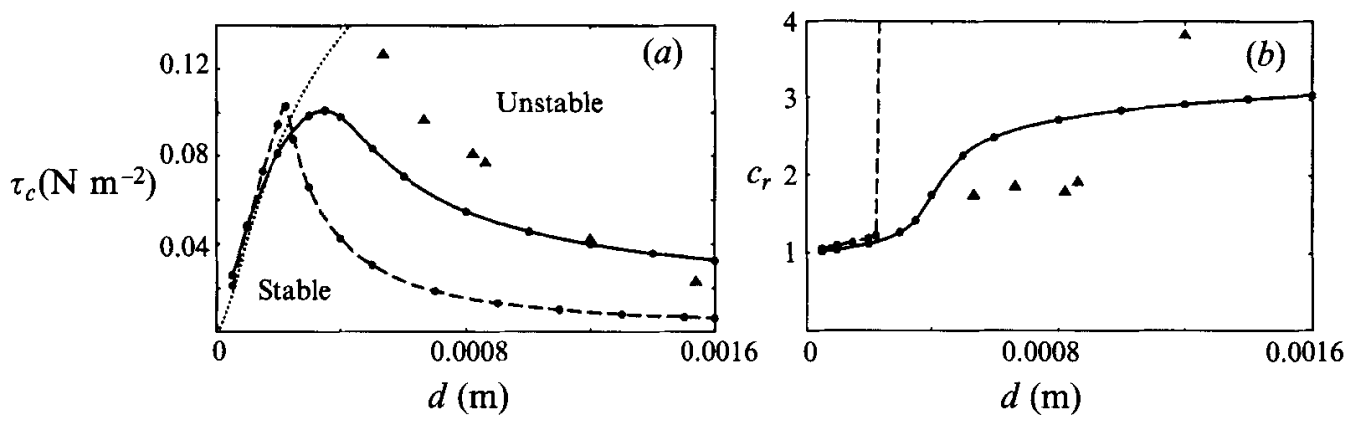

FIGURE 11. A comparison between the experimental results (triangles) for 'fast waves' in the 1 in. channel and theory. Apart from $c_{f}=0.0032$ the parameters used are as in figure 10.

solution, does not improve on this. This discrepancy can, for example, be caused by the approximation (2.7) of the gas velocity profile, by a smaller actual value of the friction coefficient $c_{f}$ (although the value $c_{f}=0.0015$ given by Craik seems rather small already), the presence of pollution on the film surface in the experiments (leading to a lower surface tension and a lower $\tau_{c}$ ), or other experimental errors.

Improved agreement is found if it is taken into account that the wavelength should be smaller than the length of Craik's apparatus and the growth rate should be large enough for waves to grow substantionally in the time they traverse the channel (see Windt 1994). The phase velocity of the slow waves compares better to the numerical solution than found from (7.1) and (7.2), the slightly lower experimental value $(c \approx 0.8)$ being due to nonlinear effects (see Craik 1965). The jump in the phase velocity is due to the dashed curve in figure 9 having two minima.

The theoretical prediction of the critical conditions for 'fast waves' is in reasonably good agreement with experiment, the points lying in the unstable part of the $\left(d, \tau_{c}\right)$ plane and close to the boundary with the stable part. The phase velocity for "fast waves' is also in reasonable agreement with experiment.

\subsection{Observing the internal mode}

The waves observed by Cohen \& Hanratty (1965) and by Craik (1966) correspond to the interfacial mode since they move faster than the maximum liquid speed (Craik's 'slow waves' also move faster if their amplitude is sufficiently small). The question arises whether there is an experimental situation for which the internal mode leads to an observable change of the flow. With this we return to a remark made in $\$ 6.2$ that if both modes are unstable (figure 7) the internal mode can, in principle, be more important owing to nonlinear effects. The experiments in Cohen \& Hanratty (1965) and Craik (1966), however, focus on conditions for which only one mode is (marginally) unstable. We will now discuss some circumstantial evidence that the internal mode may indeed be important.

If a liquid layer is sheared by a gas flow it can be unstable. The instability with the largest growth rate has a wavenumber that is order one, i.e. a wavelength that is typically one to ten times the thickness of the liquid layer. At the same time much longer waves can exist. These waves are kinematic in nature (see e.g. Whitham 1974; Jurman \& McCready 1988) in contrast to the waves studied here and are prominent in the wave spectrum found by Bruno \& McCready (1988). In the final stage of the (nonlinear) evolution of these long waves, they have the appearance of a bore and are 
called 'roll waves' (see, for example, the papers by Hanratty and co-workers: Hanratty \& Engen 1957; Hanratty \& Hershman 1961; Miya, Woodmansee \& Hanratty 1971).

These roll waves are not only an interesting wave phenomenon that can be easily observed, but are also directly related to the mechanism by which droplets are entrained from the film. This was already suggested by Hall-Taylor \& Hewitt (1962) and photographs in a report by Steen \& Wallis (1964) give the conclusive proof. More precisely, the entrainment mechanism is the formation of droplets from short waves that are sheared from the crests of roll waves. The observation that the formation of roll waves coincides with the onset of entrainment is confirmed by, for example the experiments of Andreussi, Asali \& Hanratty (1985).

For Reynolds numbers of the liquid layer that are sufficiently large $(R>400)$, the generation of roll waves can be understood in terms of the destabilizing influence of, for example, the component of the normal stress that is in phase with the wave height (Hanratty 1991, Andreussi et al. 1985). The critical Reynolds number for the gas thus found depends only weakly on the Reynolds number of the liquid and is in excellent agreement with experiments. For smaller liquid Reynolds numbers the critical gas Reynolds number for roll wave formation rapidly increases and appears to have an asymptote at a finite liquid Reynolds number $(R \approx 200$ for air-water flow). This is in contradiction with theory (Hanratty 1991), which predicts a finite critical gas Reynolds number as the liquid Reynolds number goes to zero.

The only explanation of this asymptote given so far can be found in a paper by Andreussi $e t$ al. (1985). They assume parameters, such as the friction factor, to vary along the wave and the film Reynolds number to have a relaxation time with respect to the local conditions (e.g. the thickness and velocity of the film). Owing to the fact that the friction coefficient depends on the Reynolds number this leads to an out-of-phase component of the interfacial shear stress, which stabilizes the film at low Reynolds numbers. The assumption on the relaxation of the Reynolds number is however, not clear to us. Relaxation of the friction factor, giving similar results, is easier to justify: the friction coefficient depends on the surface roughness, which is determined by the presence of short waves. These waves do, however, not respond instantaneously to the variation of the local conditions because their growth rate is finite. (A relaxation effect - a downstream maximum - of the shear stress may exist even if the surface roughness has no relaxation effect (Fredsøe et al. 1993).)

It is interesting to note that for a water-air flow the internal mode becomes unstable for a liquid Reynolds number that is also order 100 (figure 4, if the surface tension parameter $S$ is not too large). The interfacial mode on the other hand is unstable for the whole range of parameter values for which roll waves are observed. The growth of roll waves directly from the internal mode seems unlikely in view of the work of Bruno \& McCready (1988), who study the evolution of the wave spectrum of disturbances on a sheared film, and the difference in the mechanism that leads to roll waves (Hanratty 1991) and short waves (Miles 1962a). A way, however, in which the internal mode can have an effect on the formation of roll waves is through the fact that it influences the surface roughness of the film. A possibility is that the internal mode can give rise to a relaxation effect, while the interfacial mode cannot because of its much larger growth rate. An alternative way to explain the transition is "by a somewhat sudden change in the rate of increase of $f_{i}$ (the friction factor) with increasing $\operatorname{Re}_{L}$ (the liquid Reynolds number)'. This sudden increase could then be caused by the internal mode becoming unstable. A test on the validity of the above suggestions is to compare the effect that the physical properties of the liquid and the gas have on the value of the liquid Reynolds number above which roll waves 
are observed and the effect on the critical Reynolds number for the internal mode (Miesen 1995).

\section{Conclusion}

We have studied the stability of a thin liquid film that is sheared by a concurrent gas flow. We discuss the free surface approximation of the problem, in which the effect of the wave-induced fluctuations in the gas flow, i.e. the dynamics of the gas, is neglected. It is shown that the interface conditions of Miles (1960) should be used and not those of Smith \& Davis (1982). Our results for the problem in the free surface approximation resolve a discrepancy between the asymptotic solution of Miles (1960) and the numerical solution of Smith \& Davis (1982). We find a second curve of neutral stability, not noticed by Smith \& Davis, that corresponds to asymptotic result of Miles in the high Reynolds number limit $(\$ 4)$.

In the second part of the paper the dynamic effect of the gas is considered. From a comparison of the results in this case with those in the free surface approximation it is clear that the free surface approximation is, in general, not correct ( $\$ 5$ ). Including the dynamics of the gas in the problem also introduces a second mode of instability (Blennerhassett 1980; Yiantsios \& Higgins 1988), which is shown to correspond to capillary gravity waves studied by, for example, Miles (1962a) and van Gastel et al. (1985) (\$6).

In the last part $(\$ 7)$ our results are compared to the much cited experimental and theoretical results of Cohen \& Hanratty (1965) and Craik (1966). It is shown that the agreement between theory and experiment is improved if the linear stability problem is solved directly from the Orr-Sommerfeld equations for the gas and the liquid flow that are coupled by interface conditions. Finally, we discuss how the internal mode can play a role in the generation of 'roll waves', which are of great importance in describing entrainment of droplets from the film. We suggest that the critical Reynolds number for the internal mode and for roll wave generation are related.

We would like to thank $\operatorname{Dr}$ Antony Rigby for his comments on the manuscript.

\section{Appendix. Eigenvalue equation}

Expanding the determinant in equation (4.8) gives

$$
\alpha(c+1) F(z)\left(\varpi_{1 i}-2 \alpha^{2} \chi_{1 i} \frac{\varpi_{4 i}}{\chi_{4 i}^{\prime \prime}+\alpha^{2} \chi_{4 i}}\right)-\varpi_{2 i}+2 \alpha^{2} \chi_{2 i} \frac{\varpi_{4 i}}{\chi_{4 i}^{\prime \prime}+\alpha^{2} \chi_{4 i}}=0
$$

where we have used the definition (4.10), so that with (4.6)

$$
\frac{\chi_{3 w}}{\chi_{3 w}^{\prime}}=-(\alpha R)^{-1 / 3} z F(z) \text {. }
$$

Substitution of (4.4), (4.5) and (4.7) into (A 1) gives after some manipulation,

$$
\begin{aligned}
& {\left[\alpha \operatorname{coth} \alpha+\frac{\alpha^{2} \operatorname{cosech}^{2} \alpha F(z)(c+1)}{1-\alpha(c+1) F(z) \operatorname{coth} \alpha}\right](c+2 \mathrm{i} \alpha / R)\left[c+2 \mathrm{i}\left(1+\epsilon_{1}\right) \alpha / R\right]} \\
& \quad+\left[c+2 \mathrm{i}\left(1+\epsilon_{1}\right) \alpha / R\right]\left(1-2 \alpha^{2} \epsilon_{2}\right)-\alpha^{2} S\left[1+4 \alpha^{2}\left(1+\epsilon_{1}\right)^{2} /\left(c^{2} R^{2}\right)\right]=0,
\end{aligned}
$$

where $\epsilon_{1}=-2 \mathrm{e}^{\mathrm{i} \pi / 4}(\alpha R)^{-1 / 2} c^{-3 / 2}$ and $\epsilon_{2}=-2 \alpha^{2} \mathrm{e}^{-\mathrm{i} \pi / 4}(\alpha R)^{-3 / 2} c^{-1 / 2}$. Neglecting now terms of order $R^{-3 / 2}$ and of order $S / R^{2}$ gives equation (4.9). 


\section{REFERENCES}

Abramowitz, M. \& Stegun, I. A. 1965 Handbook of Mathematical Functions. Dover.

ANDReussi, P., Asali, J. C. \& HanRatTy, T. J. 1985 Initiation of roll waves in gas-liquid flow. AIChEJ. 31, 119-126.

BENJAMIN, T. B. 1959 Shearing flow over a wavy boundary. J. Fluid Mech. 6, 161-205.

BlENNERHASSETT, P. J. 1980 On the generation of waves by wind. Phil. Trans. R. Soc. A 298, 451-494.

BoOMKAMP, P. \& MiESEN, R. 1992 Nonaxisymmetric waves in core-annular flow with a small viscosity ratio. Phys. Fluids A 4, 1627-1636.

Bruno, K. \& MCCready, M. J. 1988 Origin of roll waves in horizontal gas-liquid flows. AIChEJ. 34, 1431-1440.

Cohen, L. S. \& Hanratty, T. J. 1965 Generation of waves in the concurrent flow of air and a liquid. AIChEJ. 11, 138-144.

CRAIK, A. D. D. 1965 Wind-generated waves in liquid films. PhD dissertation, University of Cambridge.

CRAIK, A. D. D. 1966 Wind-generated waves in thin liquid films. J. Fluid Mech. 26, 369-392.

Drazin, P.G. \& REID, W. H. 1986 Hydrodynamic Stability. Cambridge University Press.

DuIN, C. A. VAN \& JANSSEN, P. A. E. M. 1992 An analytical model of the generation of surface gravity waves by turbulent air flow. J. Fluid Mech. 236, 197-215.

Feldman, S. 1957 On the hydrodynamic stability of two viscous incompressible fluids in parallel uniform shearing motion. J. Fluid Mech. 2, 343-370.

Fredsøe, J., Sumer, B. M., Laursen, T. S. \& Pedersen, C. 1993 Experimental investigation of wave boundary layers with a sudden change in roughness. J. Fluid Mech. 252, 117-145.

GaSTEL, K. van, JanSSEN, P. A. E. M. \& Komen, G. J. 1985 On phase velocity and growth rate of wind-induced gravity-capillary waves. J. Fluid Mech. 161, 199-216.

HALl-TAYLOR, N. \& HeWITT, G. F. 1962 The motion and frequency of large disturbance waves in annular two-phase flow of air-water mixtures. AERE-R 3955, Harwell, UK.

HaNRaTTY, T. J. 1991 Separated flow modelling and interfacial transport phenomena. Appl. Sci. Res. 48, 353-390.

HanRatTy, T. J. \& ENGEN, J.M. 1957 Interaction between a turbulent air stream and a moving water surface. AIChEJ. 3, 299-304

HaNratTy, T. J. \& Hershman, A. 1961 Initiation of roll waves. AIChEJ. 7, 488-497.

Hetsroni, G. 1982 Handbook of Multiphase Systems. McGraw-Hill.

HiNZE, J. O. 1975 Turbulence. McGraw-Hill.

HOOPER, A. P. \& BoYD, W. G. C. 1983 Shear-flow instability at the interface between two viscous fluids. J. Fluid Mech. 128, 507-528.

HoOPER, A. P. \& BoYD, W. G. C. 1987 Shear-flow instability due to a wall and a viscosity discontinuity at the interface. $J$. Fluid Mech. 179, 201-225.

JuRman, L. A. \& MCCREady, M. J. 1989 Study of waves on thin liquid films sheared by turbulent gas flows. Phys. Fluids A 1, 522-536.

KaYs, W. M. \& CRAwford, M. E. 1966 Convective Heat and Mass Transfer. McGraw-Hill.

LiN, C.C. 1955 The Theory of Hydrodynamic Stability. Cambridge University Press.

Lock, R.C. 1954 Hydrodynamic stability of the flow in the laminar boundary layer between parallel streams. Proc. Camb. Phil. Soc. 50, 105-124.

Miesen, R. 1995 Hydrodynamic stability of liquid films. In Proc. IUTAM Symp. Waves in Liquid/Gas and Liquid/Vapor Two-Phase Systems, Kyoto, Japan, (ed. S. Morioka \& L. van Wijngaarden) pp. 245-256. Kluwer.

Miesen, R., Beijnon, G., Duijvestijn, P. E. M., Oliemans, R. V. A. \& Verheggen, T. 1992 Interfacial waves in core-annular flow. J. Fluid Mech. 238, 97-117.

MiLes, J. W. 1957 On the generation of surface waves by shear flows. J. Fluid Mech. 3, 185-204.

Miles, J. W. 1959 On the generation of surface waves by shear flows. Part 2. J. Fluid Mech. 6, $568-582$.

MiLES, J. W. 1960 The hydrodynamic stability of a thin film of liquid in uniform shearing motion. J. Fluid Mech. 8, 593-610.

Miles, J. W. $1962 a$ On the generation of surface waves by shear flows, Part 4. J. Fluid Mech. 13, 433-448. 
MILES, J. W. $1962 b$ A note on the inviscid Orr-Sommerfeld equation. J. Fluid Mech. 13, 427-432.

Miya, M., Woodmansee, D. E. \& HanRaTtY, T. J. 1971 A model for roll waves in gas-liquid flow. Chem. Engng Sci. 26, 1915-1931.

MolaR, C. B. \& StEWART, G. W. 1973 An algorithm for generalized matrix eigenvalue problems. SIAM J. Numer. Anal. 10, 241-256.

OrszaG, S. A. 1971 Accurate solution of the Orr-Sommerfeld stability equation. J. Fluid Mech. 50, 689-703.

Phillips, O. M. 1962 Resonant phenomena in gravity waves. Proc. Symp. in Appl. Maths 13, 91.

SmITH, M. K. \& DAvIS, S. H. 1982 The instability of sheared liquid layers. J. Fluid Mech. 121, 187-206.

StEen, D. A. \& WALlis, G. B. 1964 The transition from annular to annular-mist co-current two-phase downflow. U.S. Atomic Energy Commission Rep. NYO-3114-2.

VALENZUELA, G. R. 1976 The growth of gravity-capillary waves in a coupled shear flow. J. Fluid Mech. 76, 229-250.

WALLIS, G.B. 1969 One-Dimensional Two-phase Flow. McGraw-Hill.

WhithaM, G.B. 1974 Linear and Nonlinear Waves. Wiley.

WINDT, J. 1994 The influence of velocity profiles on the hydrodynamic stability of sheared liquid films. Master thesis, Twente University, Enschede, The Netherlands.

Wolfram, S. 1991 Mathematica, A System for Doing Mathematics by Computer. Addison Wesley.

YiANTSIOS, S. G. \& HigGINS, B. G. 1988 Linear stability of plane Poiseuille flow of two superposed fluids. Phys. Fluids 31, 3225-3238 and Erratum Phys. Fluids A 1, 897.

YIH, C.-S. 1967 Instability due to viscosity stratification. J. Fluid Mech. 27, 337-352.

YIH, C.-S. 1990 Wave formation on a liquid layer for de-icing airplane wings. J. Fluid Mech. 212, 41-53. 